\title{
Optical manipulation of domains in chiral topological superconductors
}

\author{
Tao Yu $\odot,{ }^{1}$ Martin Claassen, ${ }^{2,3}$ Dante M. Kennes, ${ }^{4,1}$ and Michael A. Sentef $\odot{ }^{1}$ \\ ${ }^{1}$ Max Planck Institute for the Structure and Dynamics of Matter, Luruper Chaussee 149, 22761 Hamburg, Germany \\ ${ }^{2}$ Department of Physics, University of Pennsylvania, Philadelphia, Pennsylvania 19104, USA \\ ${ }^{3}$ Center for Computational Quantum Physics, Simons Foundation Flatiron Institute, New York, New York 10010, USA \\ ${ }^{4}$ Institut für Theorie der Statistischen Physik, RWTH Aachen University and JARA-Fundamentals of Future Information Technology, \\ 52056 Aachen, Germany
}

(Received 2 October 2020; revised 5 March 2021; accepted 8 March 2021; published 18 March 2021)

\begin{abstract}
Optical control of chirality in chiral superconductors bears potential for future topological quantum computing applications. When a chiral domain is written and erased by a laser spot, the Majorana modes around the domain can be manipulated on ultrafast timescales. Here we study topological superconductors with two chiral order parameters coupled via light fields by a time-dependent real-space Ginzburg-Landau approach. Continuous optical driving, or the application of supercurrent, hybridizes the two chiral order parameters, allowing one to induce and control the superconducting state beyond what is possible in equilibrium. We show that superconductivity can even be enhanced if the mutual coupling between two order parameters is sufficiently strong. Furthermore, we demonstrate that short optical pulses with spot size larger than a critical one can overcome a counteracting diffusion effect and write, erase, or move chiral domains. Surprisingly, these domains are found to be stable, which might enable optically programmable quantum computers in the future.
\end{abstract}

DOI: 10.1103/PhysRevResearch.3.013253

\section{INTRODUCTION}

Chiral superconductors spontaneously break time-reversal symmetry and host topologically protected chiral Majorana edge modes [1,2], thus bearing potential for applications in quantum computing $[3,4]$. The superconductor's chirality itself can be a useful degree of freedom, much like spin $[5,6]$, valley [7,8], and other quantum states [9], which may allow for quantum information processing [10]. It is therefore desirable to develop means of controlling chiral superconductors and their associated Majorana edge modes, preferably on ultrafast timescales. Dynamical symmetry breaking by optical driving [10-12], or supercurrents [13,14], has been suggested as an efficient way to achieve this goal.

It was recently predicted that optical switching of chirality in a bulk chiral superconductor can be achieved by a joint effect of homogeneous linearly and circularly polarized optical pulses [10]. The local and ultrafast manipulation of Majorana modes, however, requires the creation and annihilation of chiral domains by a laser spot of finite size. It is not $a$ priori obvious that this can be achieved since locally perturbed order parameters can diffusively relax back to their original state in a system with multicomponent superconducting instabilities. Here we microscopically derive the time-dependent

Published by the American Physical Society under the terms of the Creative Commons Attribution 4.0 International license. Further distribution of this work must maintain attribution to the author(s) and the published article's title, journal citation, and DOI. Open access publication funded by the Max Planck Society.
Ginzburg-Landau (TDGL) equations [15,16] in real space from a prototype model on a honeycomb lattice [17-21], which could be realized, as suggested by recent experiments, in highly doped graphene [22,23] or twisted bilayer graphene and other van der Waals materials [24-32]. Further works on chiral superconducting materials, which will follow a similar phenomenology as discussed here, include experimental evidence for chiral $p \pm i p$-wave superconductivity in materials, such as $\mathrm{UPt}_{3}$ [33-35] and $\mathrm{UTe}_{2}$ [36]. The perhaps most notable candidate material has been $\mathrm{Sr}_{2} \mathrm{RuO}_{4}$ with proposals of triplet $p$-wave pairing $[37,38]$, but a more recent investigation ruled this out [39], and there is now evidence for $d$-or potentially even $g$-wave instabilities [40-42]. Importantly, our TDGL theory phenomenologically but transparently describes the dynamics of coupled order parameters of general chiral superconductors in both spatially homogeneous and inhomogeneous scenarios.

Our main results are twofold. On the one hand, we find that because the two chiral order parameters are coupled via the optical vector potential as a consequence of angularmomentum conservation, a continuous driving (or a static supercurrent) can hybridize the two order parameters. Provided that the coupling between the two order parameters is sufficiently strong, superconductivity can even be enhanced beyond their equilibrium values. On the other hand, we demonstrate that a short pulse of linearly polarized light can drive the two order parameters to be close in magnitude. Due to the accompanying amplitude-mode oscillation (Higgs mode) [43-50], the balance between the two chiral order parameters depends on the precise time at which the pulse is switched off with respect to this oscillation. The probability for a switch from one order to the other to occur is roughly 

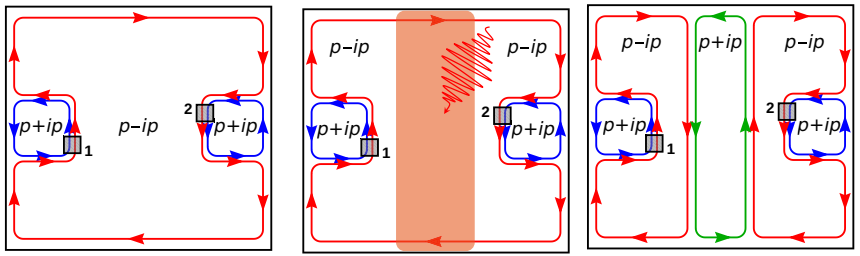

FIG. 1. Interference of Majorana fermions using optically engineered domains in a $p+i p$ superconductor. A single electron is injected at the two leads (gray shaded boxes) into the domain walls between the $p+i p$ and $p-i p$ states, which carry two copropagating Majorana modes. Depending on the topography of the domains, the fractionalization and propagation of these electrons along the Majorana boundary modes implements either a Hadamard gate (left) or an identity operation (right) on the charging states of the leads. Using optical manipulation, one can switch between these two gates on ultrafast timescales (center).

$1 / 2$. A second circularly polarized pulse can be used to favor one chiral order parameter over the other in order to achieve a reliable switching [10]. Finally, for the spatially inhomogeneous situation, we predict that there is a minimal spot size required to overcome the diffusion effect, which counteracts the switching. Above a critical spot, size switching can be obtained in a stable fashion, allowing for the optical creation or annihilation of chiral domains of multicomponent superconductors $[10,21]$, such as the widely studied chiral $p \pm i p$ superconductivity. A combination of creation and annihilation can be used to move the domain.

Since the induced chiral domain is stable after application of optical pulses, manipulation of Majorana modes at the boundary of such domains is possible. This could be used to optically program quantum logic gates. Figure 1 depicts a possible implementation of a Hadamard gate [51-53] using the Majorana modes of optically engineered domains in a $p+i p$ superconductor. Analogous to the mechanism described in Ref. [4], the single-electron charging states of two leads can be used to encode quantum bits of information [10]. Upon injection of a single electron at a $p+i p / p-i p$ domain-wall boundary that carries a pair of Majorana modes or, equivalently, a single chiral complex fermion mode, the electron can propagate along the edge channel. Crucially, the superconducting sample boundary hosts only a single Majorana mode, necessitating a fractionalization of the electron into Majorana fermions. By an optically induced choice of domain topography, these propagating Majorana modes can then implement interference, realizing a Hadamard gate on the charging states of the leads [4].

Importantly, we need to go beyond Ref. [10] to investigate the spatial-temporal dynamics that is required in such a device. To this end, we explicitly formulate inhomogeneous scenarios by Ginzburg-Landau phenomenology. Our theory transparently describes the coupling of order parameter components via an optical field, addresses the conditions of optical enhancement of chiral superconductivity, reveals the necessary conditions for local switching of chirality, and elevates the concept introduced in Ref. [10] towards the functionalities of quantum gates.



FIG. 2. Optical engineering of chiral superconductivity on a honeycomb lattice. Geometrical parameters are indicated: $\mathbf{b}_{\mu=1,2,3}$ are three bonding vectors connecting the $B$ and $A$ lattices, which define the two primitive vectors of the lattice by $\mathbf{a}_{1}=\mathbf{b}_{1}-\mathbf{b}_{3}$ and $\mathbf{a}_{2}=$ $\mathbf{b}_{2}-\mathbf{b}_{3}$. An in-plane optical driving is applied, as indicated by the red arrow.

\section{MODEL AND FORMALISM}

To be specific, we consider a chiral $d \pm i d$ superconductor on a honeycomb lattice, as illustrated in Fig. 2. The general results, however, will carry over to other chiral superconductors (with two-component order parameters), such as $p \pm i p$ superconductors. The tight-binding $t-J$ model Hamiltonian reads [17-21]

$$
\begin{aligned}
\hat{H}_{t-J}= & -t \sum_{\langle i, j\rangle, \sigma}\left(\hat{a}_{i \sigma}^{\dagger} \hat{b}_{j \sigma}+\hat{b}_{j \sigma}^{\dagger} \hat{a}_{i \sigma}\right)-\mu \sum_{i, \sigma}\left(\hat{a}_{i \sigma}^{\dagger} \hat{a}_{i \sigma}+\hat{b}_{i \sigma}^{\dagger} \hat{b}_{i \sigma}\right) \\
& -J \sum_{\langle i, j\rangle} \hat{h}_{i j}^{\dagger} \hat{h}_{i j},
\end{aligned}
$$

with $\hat{a}_{\sigma}$ and $\hat{b}_{\sigma}$ being the annihilation operators of electrons with spin $\sigma=\{\uparrow, \downarrow\}$ on the $A$ and $B$ sublattices. The hopping amplitude between nearest-neighbor lattice sites is denoted by $t$. The system is assumed to be doped near the van Hove singularity with the chemical potential $\mu \sim t[17,18,22-29]$. We consider a nearest-neighbor spin exchange interaction $J$ between spin singlets with creation operators $\hat{h}_{i j}^{\dagger}=$ $\left(\hat{a}_{i \uparrow}^{\dagger} \hat{b}_{j \downarrow}^{\dagger}-\hat{a}_{i \downarrow}^{\dagger} \hat{b}_{j \uparrow}^{\dagger}\right)$ with sites $i \in A$ and $j \in B[17,18]$. Based on this Hamiltonian, we employ the path-integral technique, with the introduction of three boson fields at the three bondings $\mathbf{b}_{\mu}$ by the Hubbard-Stratonovich transformation [54], to set up the Ginzburg-Landau (GL) Lagrangian for the superconducting order parameters [54] (see Appendix A for detailed derivations).

With the order parameters $\eta_{1}(\mathbf{r}, t), \eta_{2}(\mathbf{r}, t)$ of the $\left(d_{x^{2}-y^{2}}+\right.$ $\left.i d_{x y}\right)-,\left(d_{x^{2}-y^{2}}-i d_{x y}\right)$-superconducting tendency, respectively, the GL Lagrangian density reads

$$
\begin{aligned}
\mathcal{L}_{\mathrm{eff}}(\mathbf{r})= & \sum_{\mu=1,2} \Gamma_{\mu} \eta_{\mu}^{*}(\mathbf{r}) D_{t} \eta_{\mu}(\mathbf{r})+\sum_{\mu} \Lambda_{\mu}\left|D_{t} \eta_{\mu}(\mathbf{r})\right|^{2} \\
& +a \sum_{\mu}\left|\eta_{\mu}(\mathbf{r})\right|^{2}+b \sum_{\alpha=x, y} \sum_{\mu}\left|D_{\alpha} \eta_{\mu}(\mathbf{r})\right|^{2} \\
& +e_{1}(1-i \sqrt{3})\left[D_{+} \eta_{2}^{*}(\mathbf{r})\right]\left[D_{+} \eta_{1}(\mathbf{r})\right]
\end{aligned}
$$




$$
\begin{aligned}
& +e_{1}(1+i \sqrt{3})\left[D_{+}^{*} \eta_{2}(\mathbf{r})\right]\left[D_{+}^{*} \eta_{1}^{*}(\mathbf{r})\right] \\
& +f_{1}\left(\left|\eta_{1}\right|^{2}+\left|\eta_{2}\right|^{2}\right)^{2}+f_{2}\left(\left|\eta_{1}\right|^{2}-\left|\eta_{2}\right|^{2}\right)^{2} .
\end{aligned}
$$

The $s$-wave order parameter is disregarded here, as it is not energetically favored $[10,17,18]$ and does not affect the main conclusions presented here (its inclusion is analyzed in Appendices A and B). Here, $D_{t} \equiv \partial_{t}-(2 e / i \hbar) \varphi(\mathbf{r}, t)$, $D_{\alpha}=\partial_{\alpha}-(2 e / i \hbar c) \mathbf{A}_{\alpha}(\mathbf{r}, t)$, and $D_{ \pm}=D_{x} \pm i D_{y}$ are the covariant derivatives that respect the gauge invariance of the Lagrangian [21], where $\varphi(\mathbf{r}, t)$ and $\mathbf{A}(\mathbf{r}, t)$ are the scalar and vector potentials of the electromagnetic field, and $c$ is the speed of light. The first $\Gamma$ term is of the Gross-Pitaevskii type, and is responsible for dissipation $[15,16]$ back to equilibrium. The second $\Lambda$ term is of the Klein-Gordon type, which leads to collective-mode dynamics, such as the amplitude or Higgs mode, that is defined by the fluctuation of the order-parameter amplitude $[43,48,50]$. Here the parameters $\Gamma_{\mu}$ and $\Lambda_{\mu}$ are treated phenomenologically and are allowed to be different for the two $d \pm i d$ order parameters only if time-reversal symmetry is explicitly broken, for instance by a circularly polarized laser field, following Ref. [10]. The other coefficients $\left\{a<0, b, e_{1}, f_{1}>0, f_{2}=\right.$ $\left.-f_{1} / 3\right\}$ are all real numbers that are microscopically calculated through the Hamiltonian (1); see Appendix A. Estimated by graphene's material parameters $t=2.7 \mathrm{eV}$, the bonding length $|\mathbf{b}|=2.46 / \sqrt{3} \AA$ [55], $\mu=0.9 t$, and $J=0.25 t$ $[10,18], a=-10^{-3} /|\mathbf{b}|^{2} \mathrm{meV}^{-1} \mathrm{~m}^{-2}, b=800 \mathrm{meV}^{-1}, e_{1}=$ $150 \mathrm{meV}^{-1}$, and $f_{1}=2.1 /|\mathbf{b}|^{2} \mathrm{meV}^{-3} \mathrm{~m}^{-2}$ at $T=1.5 \mathrm{~K}$. These parameters correspond to a critical temperature $T_{c}=$ $4.7 \mathrm{~K}$. We choose $\Gamma=5|a| \hbar /\left(k_{B} T_{c}\right)$, with a realistic nanosecond relaxation time for small order-parameter fluctuations, and $\Lambda=-a \hbar^{2} /\left(2\left|\eta_{0}\right|^{2}\right)$, with the superconducting gap $\eta_{0}=$ $\sqrt{-a /\left(2 f_{1}+2 f_{2}\right)}=0.02 \mathrm{meV}$, following Ref. [16]. However, importantly, this GL Lagrangian can also be constructed based entirely on symmetry considerations [21], independent of microscopic details, rendering our results applicable to a wide range of physical situations.

When $f_{2}<0$, the two chiral $(d \pm i d)$ waves are degenerate superconducting ground states $[17,18]$, which is the case for Hamiltonian (1) (see Appendix B). Otherwise, the $d_{x^{2}-y^{2}}=$ $\left(\eta_{1}+i \eta_{2}\right) / 2$ and $d_{x y}=\left(\eta_{1}-i \eta_{2}\right) / 2$ waves are the ground states. As the two order parameters $\eta_{1}$ and $\eta_{2}$ are coupled in the $e_{1}$ terms, obeying angular-momentum conservation, their interplay can be optically controlled. From $\delta L / \delta \eta_{1,2}^{*}(\mathbf{r})=0$ [15], we obtain the TDGL equations

$$
\begin{aligned}
& \left(\begin{array}{cc}
\Gamma_{1} & 0 \\
0 & \Gamma_{2}
\end{array}\right) D_{t}\left(\begin{array}{l}
\eta_{1} \\
\eta_{2}
\end{array}\right)+\left(\begin{array}{cc}
\Lambda_{1} & 0 \\
0 & \Lambda_{2}
\end{array}\right) D_{t}^{*} D_{t}\left(\begin{array}{l}
\eta_{1} \\
\eta_{2}
\end{array}\right) \\
& +\left(\begin{array}{cc}
a-b \sum_{\alpha=x, y} D_{\alpha}^{2} & -e_{1}(1+i \sqrt{3}) \tilde{D}_{-} D_{-} \\
-e_{1}(1-i \sqrt{3}) \tilde{D}_{+} D_{+} & a-b \sum_{\alpha=x, y} D_{\alpha}^{2}
\end{array}\right)\left(\begin{array}{l}
\eta_{1} \\
\eta_{2}
\end{array}\right) \\
& +\left(\begin{array}{cc}
\mathcal{F}_{+}\left(\left|\eta_{1}\right|^{2},\left|\eta_{2}\right|^{2}\right) & 0 \\
0 & \mathcal{F}_{-}\left(\left|\eta_{1}\right|^{2},\left|\eta_{2}\right|^{2}\right)
\end{array}\right)\left(\begin{array}{l}
\eta_{1} \\
\eta_{2}
\end{array}\right)=0,
\end{aligned}
$$

where $\quad \tilde{D}_{v}=\partial_{v}+(2 e / i \hbar c) \mathbf{A}_{v}(\mathbf{r}), \quad \tilde{D}_{ \pm}=\tilde{D}_{x} \pm i \tilde{D}_{y}, \quad$ and $\mathcal{F}_{ \pm}\left(\left|\eta_{1}\right|^{2},\left|\eta_{2}\right|^{2}\right) \equiv 2 f_{1}\left(\left|\eta_{1}\right|^{2}+\left|\eta_{2}\right|^{2}\right) \pm 2 f_{2}\left(\left|\eta_{1}\right|^{2}-\left|\eta_{2}\right|^{2}\right)$. We choose a gauge with $\varphi=0$ and consider the dynamics under vector potentials $\mathbf{A}_{x, y}(\mathbf{r}, t)$ that are, in general, spatially and temporally dependent, which we shall specify in the following.
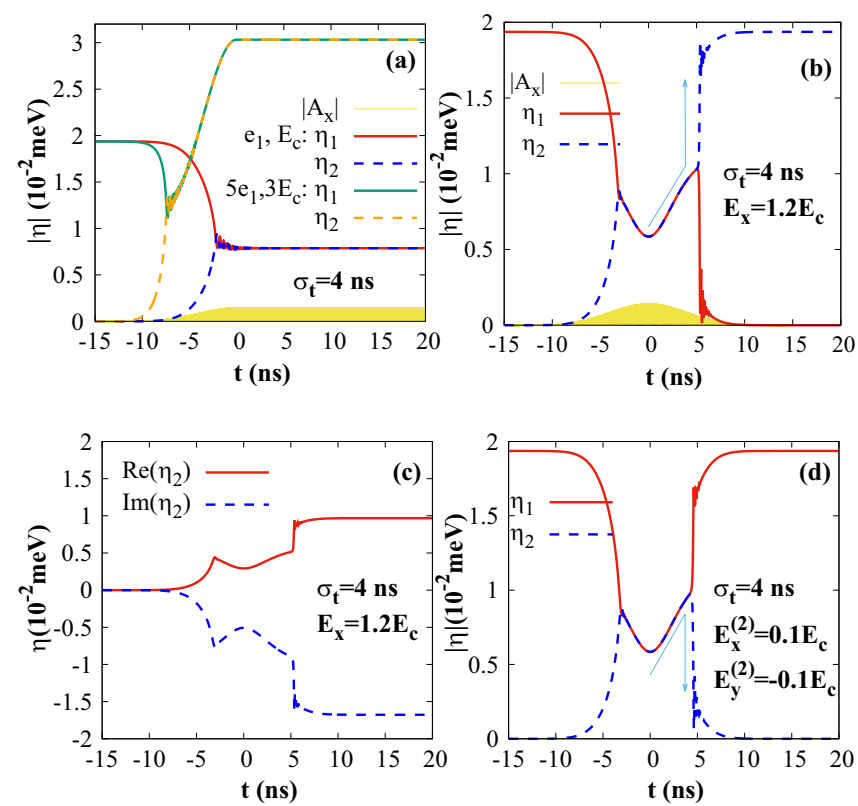

FIG. 3. Optical engineering of order parameters by homogeneous optical fields. (a) Hybridization of two chiral order parameters by continuous linearly polarized optical fields. An arbitrary value $5 e_{1}$ for the order-parameter coupling is used for illustration of superconductivity enhancement. Optical switching of chirality is shown in (b) by order parameter magnitude and (c) by real and imaginary components of $\eta_{2}$. In (d), a second left-circularly polarized laser with time delay $\sigma_{t}$ relative to the first linearly polarized laser is applied to reverse the chirality, while the right-circularly polarized laser can retain the chirality (not shown). Parameters used for calculation are given in the figure and text.

\section{MECHANISM OF CHIRALITY SWITCHING}

We first discuss the coupling between the two chiral order parameters through light in the homogeneous case. Here, we can analyze the chirality switching mechanism analytically. The coupling of the two order parameters is $e_{1}\left(\mathbf{A}_{x}^{2}-\mathbf{A}_{y}^{2}\right) \rightarrow$ 0 for the circularly polarized laser within the rotating-wave approximation. Therefore, as also confirmed by our numerical calculation, circularly polarized light by itself cannot cause chirality switching. We therefore focus here on the linearly polarized laser first.

We first analyze the hybridization of order parameters by a continuous optical driving of frequency $\omega$ and electricfield amplitude $\mathbf{E}$, say, $\mathbf{A}_{x}=-(c / \omega) \mathbf{E}_{x} \cos (\omega t)$ (equivalent to sourcing a constant supercurrent). When the two chiral order parameters are driven to the same magnitude, we use the ansatz $\tilde{\eta}_{2}=\tilde{\eta}_{1} e^{i \phi}$ to solve the TDGL equation (3) and find $\phi=-\pi / 3$ and magnitude

$$
\left|\tilde{\eta}_{1,2}\right|=\sqrt{\frac{1}{4 f_{1}}\left[-a+2\left(2 e_{1}-b\right)\left(\frac{e E_{x}}{\hbar \omega}\right)^{2}\right]},
$$

which is tunable by the field strength. This implies that by driving, superconductivity is suppressed when $2 e_{1}<b$, otherwise it is enhanced due to the strong spatial fluctuation between the two order parameters. Such a hybridization is confirmed in Fig. 3(a) with adiabatically turned on con- 
tinuous driving of frequency $\hbar \omega=20 \mathrm{meV}$ with $\mathbf{A}_{x}=$ $-(c / \omega) \mathbf{E}_{x} \cos (\omega t) \exp \left[-t^{2} /\left(2 \sigma_{t}^{2}\right)\right]$ for duration time $\sigma_{t}=$ 4 ns for $t<0$ and $-(c / \omega) \mathbf{E}_{x} \cos (\omega t)$ for $t>0$. This hybridization also implies the existence of a critical supercurrent that induces nodes in the superconducting gap before superconductivity is destroyed, providing a unique feature of chiral superconductors.

We now turn to the discussion of chirality switching with short pulses in the homogeneous case. The similar magnitude of order parameters through driving is essential for the chirality switching, which happens when the passive order parameter, initially absent, overcomes the initially active one. The order parameters can be driven to be close only when the field strength is larger than a critical value $E_{c} \approx(\hbar \omega / e) \sqrt{-a /\left(b-2 e_{1}\right)}=1 \mathrm{kV} / \mathrm{cm}$, estimated by setting $\left|\tilde{\eta}_{1,2}\right| \rightarrow \eta_{0} / 2$ in Eq. (4). Figure 3(b) confirms that when the two chiral order parameters are driven to be close by a linearly polarized laser with $E_{x}=1.2 E_{c}$, switching of chirality happens after the pulse, accompanied by an oscillation of the order parameters. This indicates that the Higgs mode activation causes a temporal fluctuation that provides an opportunity for the passive order parameter to overcome its inertia. The final state $\eta_{2}$ after the switching has a phase shift $-\pi / 3$ relative to the initial one $\eta_{1}$ [Fig. 3(c)], as expected from order-parameter hybridization [Eq. (4)]. Nevertheless, when we further increase the field strength or change the laser pulse duration, the switching does not always happen. Half of the time the order parameter simply relaxes back to the initial state with no switching, which we demonstrate in thousands of calculated cases with different switch-off parameters.

An explicit breaking of time-reversal symmetry by a second circularly polarized laser, with time delay $\sigma_{t}$ to the first one, turns out to be key to obtain full control of the switching process [10]. Although the circularly polarized light cannot couple the two order parameters directly, as discussed above, it may influence their damping since single-particle excitations or the environment could be polarized by the laser [56,57]. Neither of these effects are directly included in the TDGL framework, but require a more microscopic treatment, such as Bogoliubov-de Gennes equations [10]. We therefore assume that the damping rate depends on the chirality of the laser by a minimal phenomenological ansatz $\Gamma_{1} \rightarrow \Gamma_{1}(1+$ $\left.\delta_{c} E_{x}^{(2)} E_{y}^{(2)} / E_{c}^{2}\right)$ and $\Gamma_{2} \rightarrow \Gamma_{2}\left(1-\delta_{c} E_{x}^{(2)} E_{y}^{(2)} / E_{c}^{2}\right)$, where a tiny dimensionless $\delta_{c} \sim 0.1>0$ is used. We indeed realize complete control of the switching by the chirality of the second laser pulse with this ansatz by using our parameters, recovering the discovery of Ref. [10], as shown in Fig. 3(d) for weak fields $E_{x}^{(2)}=-E_{y}^{(2)} \sim 0.1 E_{c}$ of left-handed circularly polarized light.

\section{OPTICAL ENGINEERING OF CHIRAL DOMAIN}

We now address finite-spot-sized laser pulses in order to investigate the possibility of writing, erasing, or moving chiral domains for implementations in potential future quantum computers (Fig. 1; see Appendix C for implementing Hadamard gates using Majorana edge modes). We now restrict the laser field to a spot of size $\sqrt{2} \sigma_{r}$ by using fields $\mathbf{A}_{y}(t)=$ $-(c / \omega) \mathbf{E}_{y} \sin (\omega t) \exp \left[-t^{2} /\left(2 \sigma_{t}^{2}\right)\right] \exp \left[-r^{2} /\left(2 \sigma_{r}^{2}\right)\right]$. With a
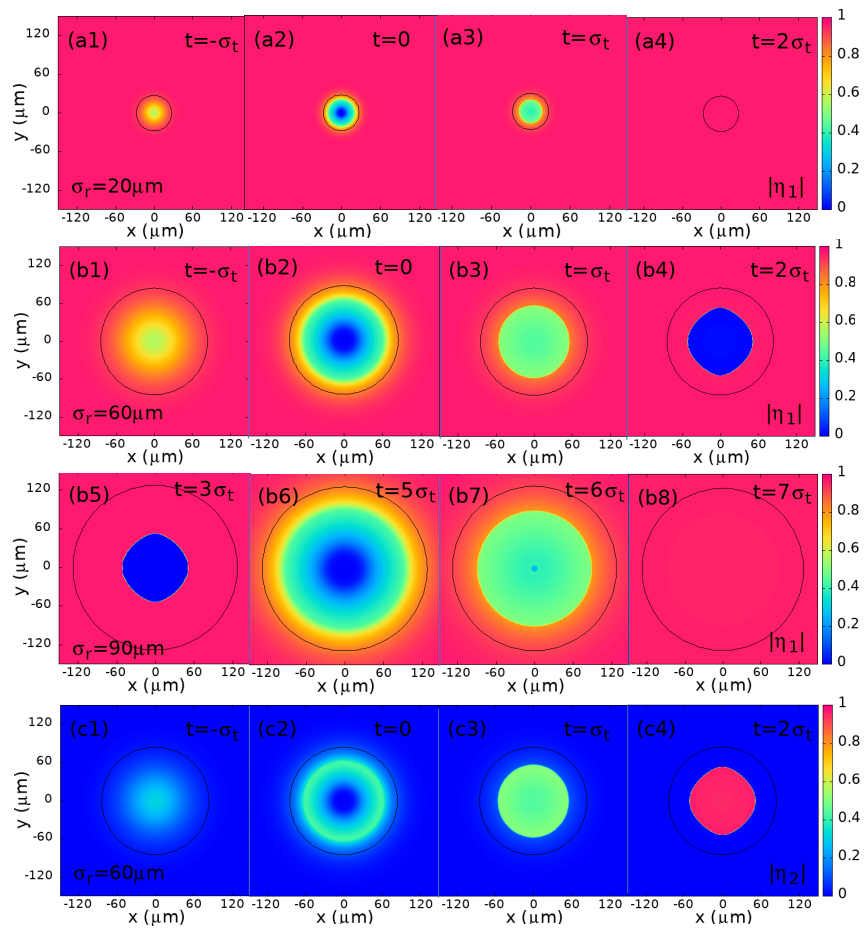

FIG. 4. Optical engineering of order parameters (normalized by $\eta_{0}$ ) in real space by laser spots. The spot size $\sqrt{2} \sigma_{r}$ is indicated by black circles. (a1)-(a4) plot the snapshots of $\left|\eta_{1}\right|$ under a laser of small size with $\sigma_{r}=20 \mu \mathrm{m}$. The order parameter relaxes to its equilibrium after the pulse [(a4)], not switched. (b1)-(b4) and (c1)(c4) plot the evolutions of $\left|\eta_{1}\right|$ and $\left|\eta_{2}\right|$, respectively, when the spot size is larger, with $\sigma_{r}=60 \mu \mathrm{m}$. Switching is achieved [(b4),(c4)] and stable afterwards. (b5) and (b6) illustrate the optical erasure of a chiral domain in real space by a linearly polarized laser at $t \in\left[4 \sigma_{t}, 6 \sigma_{t}\right]$, with spot size $\sigma_{r}=90 \mu \mathrm{m}$, and a second pulse of left-circular polarization at $t \in\left[5 \sigma_{t}, 7 \sigma_{t}\right]$, which favors the switching from $\eta_{2}$ to $\eta_{1}$. Parameters for the calculation are given in the text.

right-circularly polarized laser $E_{x}^{(2)}=E_{y}^{(2)}=0.1 E_{c}$ of $\sigma_{t}=$ 2 ns delay to this linearly polarized field $E_{y}=1.5 E_{c}$, we compute the superconductor dynamics with the TDGL equations in real space. We illustrate the results with two representative spot sizes (indicated by the black circles) $\sqrt{2} \sigma_{r}=$ $20 \sqrt{2}$ and $60 \sqrt{2} \mu \mathrm{m}$ in Fig. 4. These spot sizes are much larger than the coherence length of the order parameters, $\xi \sim \sqrt{-b /(2 a)} \approx 0.1 \mu \mathrm{m}[58]$. When the spot size is small $\left(\sigma_{r}=20 \mu \mathrm{m}\right)$, switching is not achieved after the pulse, as shown in Figs. 4(a1)-4(a4). The switching becomes possible, however, when the spot size is larger $\left(\sigma_{r}=60 \mu \mathrm{m}\right)$, as shown in Figs. 4(b4) and 4(c4).

In fact, as long as the spot size $\sigma_{r} \gtrsim 20 \mu \mathrm{m}$, we always find the chirality switching with the domain size smaller than the spot size, indicating the existence of a critical size $\sigma_{r}^{(c)}$ of photo-induced chiral domain. The switching is always reversed when the handedness of second pulse is reversed. From the TDGL equations, an order-parameter fluctuation relaxes to equilibrium on a timescale $\tau \sim 3 \mathrm{~ns}$ [compare Fig. 3(b)]; on the other hand, the excited spatial fluctuation propagates with the Higgs-mode propagation speed $v=\sqrt{\Lambda / b} \sim 5.3 \mathrm{~km} / \mathrm{s}$. 
We may thereby estimate the critical spot size as $\sigma_{r}^{(c)} \sim v \tau=$ $16 \mu \mathrm{m}$, reasonably agreeing with the numerical calculation. It is important to note that this critical spot size is two orders of magnitude larger than the superconducting coherence length in our model.

The ability of optical erasure of a chiral domain is essential for application since the combination of creation and annihilation can move a chiral domain on demand, potentially allowing for the interference of Majorana modes [2,3,51-53]. On the basis of the chiral domain in Figs. 4(b4) and 4(c4), we now apply similar optical pulses but use a left-circularly polarized laser that favors the switching from $\eta_{2}$ to $\eta_{1}$. The diffusion of order parameters at the edge leaves a ring of chiral domain with a small size (see Fig. 6 of Appendix B), which, however, can be entirely erased by pulses of larger size, such as $\sigma_{r}=90 \mu \mathrm{m}$, shown in Figs. 4(b5)-4(b8).

Again, in real space, the phase of $\eta_{2}$ has a shift $-\pi / 3$ with respect to $\eta_{1}$, and hence the order parameter at the center of the domain wall is neither $d_{x^{2}-y^{2}}$ nor $d_{x y}$, but a superposition. The free energy is increased, as the domain wall costs additional energy (see Fig. 5 in Appendix B), indicating that the chiral domain may be energetically metastable. However, the chiral order parameters carry opposite angular momenta and topological winding numbers such that their direct conversion breaks angular-momentum conservation, seemingly not possible in the absence of magnetic fields $[59,60]$. Indeed, the photo-induced chiral domain appears to be stable since it does not vanish after the pulse in the TDGL computation, robust to order-parameter fluctuation.

\section{DISCUSSION AND CONCLUSION}

Phenomenological GL approaches solely rely on system symmetry and hence do not strongly depend on the underlying mechanism for chiral superconductivity [21]. The parameters therein depend on the environment such as the substrate and phonons, which may play a role in the superconducting mechanism $[61,62]$ and, as bosonic and fermionic baths, can influence the switching processes. On the one hand, they can relax hot quasiparticles to suppress heating effects. On the other hand, they can enhance the damping rate $\Gamma$
$[10,15]$ and therefore tend to decelerate the chirality switching and favor a larger critical size of the laser spots for local switching.

We have demonstrated local control of chirality by focused laser pulses that can write, erase, and move chiral domains, and addressed the way to realize optically programmable quantum logic gates. For realistic unconventional superconductors, the required field strengths are within reach: a field of one to several $\mathrm{kV} / \mathrm{cm}$ of tens of terahertz is large enough to cause sufficient coupling between order parameters and trigger the switching, such that heating effects might be small. The required minimal size of the laser spot is tens of micrometers to overcome the diffusion when writing a chiral domain. We find that an enhancement of superconductivity may be possible in particular materials with sufficiently strong coupling of order parameters by optical driving. The hybridization of chiral order parameters is measurable by a scanning tunneling microscope, which can track the nodes in the gap that are created in this case [63]. The local chirality of the superconductor can be measured via Kerr rotation with spatial resolution $[64,65]$ or via the anomalous Hall effect [66-69]. Our study also suggests that unique features in the optical response of chiral superconductors may be useful for identifying the superconducting state of magic-angle twisted bilayer graphene and other van der Waals materials [24-32].

\section{ACKNOWLEDGMENTS}

T.Y. thanks Damian Hofmann for help on highperformance computing. T.Y. and M.A.S. acknowledge financial support by Deutsche Forschungsgemeinschaft through the Emmy Noether program (Grant No. SE 2558/2-1). The Flatiron Institute is a Division of the Simons Foundation. D.M.K. acknowledges support by the Deutsche Forschungsgemeinschaft (DFG, German Research Foundation) via RTG 1995 and Germany's Excellence Strategy - Cluster of Excellence Matter and Light for Quantum Computing (ML4Q) EXC 2004/1 - 390534769. We acknowledge support from the Max Planck-New York City Center for Non-Equilibrium Quantum Phenomena.

\section{APPENDIX A: EFFECTIVE LAGRANGIAN}

Based on the lattice model [Eq. (1)] of the honeycomb lattice (Fig. 2) [18-21], we define the continuous field operators

$$
\hat{\psi}_{A}(\mathbf{r})=\frac{1}{\sqrt{S}} \sum_{\mathbf{k}} \hat{a}(\mathbf{k}) e^{i \mathbf{k} \cdot \mathbf{r}}, \quad \hat{\psi}_{B}(\mathbf{r})=\frac{1}{\sqrt{S}} \sum_{\mathbf{k}} \hat{b}(\mathbf{k}) e^{i \mathbf{k} \cdot \mathbf{r}},
$$

where $S=N \Omega$ is the area of $N$ unit cells, with $\Omega=\sqrt{3} a^{2} / 2=3 \sqrt{3} b^{2} / 2$ being the area of one unit cell. Here, $a=\left|\mathbf{a}_{1}\right|=\left|\mathbf{a}_{2}\right|$ and $b=\left|\mathbf{b}_{\mu=\{1,2,3\}}\right|$, introduced in Fig. 2. In terms of the field operator $\hat{\Psi}(\mathbf{r})=\left[\hat{\psi}_{A \uparrow}(\mathbf{r}), \hat{\psi}_{B \uparrow}(\mathbf{r}), \hat{\psi}_{A \downarrow}^{\dagger}(\mathbf{r}), \hat{\psi}_{B \downarrow}^{\dagger}(\mathbf{r})\right]^{T}$ in the Nambu space, the free part of the Hamiltonian,

$$
\hat{H}_{0}=\int d \mathbf{r} \hat{\Psi}^{\dagger}\left(\begin{array}{cccc}
-\mu & -t f(\hat{\mathbf{k}}) & 0 & 0 \\
-t f^{\dagger}(\hat{\mathbf{k}}) & -\mu & 0 & 0 \\
0 & 0 & \mu & t f^{\dagger}(-\hat{\mathbf{k}}) \\
0 & 0 & t f(-\hat{\mathbf{k}}) & \mu
\end{array}\right) \hat{\Psi}
$$


where $f(\mathbf{k})=\sum_{\mu} e^{i \mathbf{k} \cdot \mathbf{b}_{\mu}}$, while the interaction Hamiltonian,

$$
\hat{H}_{\mathrm{int}}=-J \Omega \sum_{\mu} \int d \mathbf{r}\left[\hat{\Psi}^{\dagger}\left(\mathbf{r}_{\mu}\right) \tau_{A} \hat{\Psi}(\mathbf{r})+\hat{\Psi}^{\dagger}(\mathbf{r}) \tau_{B} \hat{\Psi}\left(\mathbf{r}_{\mu}\right)\right]\left[\hat{\Psi}^{\dagger}(\mathbf{r}) \tau_{A}^{T} \hat{\Psi}\left(\mathbf{r}_{\mu}\right)+\hat{\Psi}^{\dagger}\left(\mathbf{r}_{\mu}\right) \tau_{B}^{T} \hat{\Psi}(\mathbf{r})\right],
$$

with $\mathbf{r}_{\mu}=\mathbf{r}+\mathbf{b}_{\mu}$ and

$$
\tau_{A}=\left(\begin{array}{cccc}
0 & 0 & 0 & 0 \\
0 & 0 & 1 & 0 \\
0 & 0 & 0 & 0 \\
0 & 0 & 0 & 0
\end{array}\right), \quad \tau_{B}=\left(\begin{array}{cccc}
0 & 0 & 0 & 1 \\
0 & 0 & 0 & 0 \\
0 & 0 & 0 & 0 \\
0 & 0 & 0 & 0
\end{array}\right)
$$

Accordingly, with the Grassmann field $\bar{\Psi}(\mathbf{r}, \tau)=\left[\bar{\psi}_{A \uparrow}(\mathbf{r}), \bar{\psi}_{B \uparrow}(\mathbf{r}), \psi_{A \downarrow}(\mathbf{r}), \psi_{B \downarrow}(\mathbf{r})\right]$, the action reads

$$
\begin{aligned}
\mathcal{S}= & \int_{0}^{\beta} d \tau d \mathbf{r} \bar{\Psi} \partial_{\tau} \Psi+\int_{0}^{\beta} d \tau d \mathbf{r} \bar{\Psi}(\mathbf{r}, \tau) H_{0}(\hat{\mathbf{k}}) \Psi(\mathbf{r}, \tau)-J \Omega \sum_{\mu} \int_{0}^{\beta} d \tau d \mathbf{r}\left[\bar{\Psi}\left(\mathbf{r}_{\mu}\right) \tau_{A} \Psi(\mathbf{r})+\bar{\Psi}(\mathbf{r}) \tau_{B} \Psi\left(\mathbf{r}_{\mu}\right)\right] \\
& \times\left[\bar{\Psi}(\mathbf{r}) \tau_{A}^{T} \Psi\left(\mathbf{r}_{\mu}\right)+\bar{\Psi}\left(\mathbf{r}_{\mu}\right) \tau_{B}^{T} \Psi(\mathbf{r})\right] .
\end{aligned}
$$

We introduce the complex Bose fields $\phi\left(\mathbf{r}, \mathbf{r}_{\mu}\right)$ by Hubbard-Stratonovich transformation [54],

$$
1=\int \mathcal{D} \phi \mathcal{D} \bar{\phi} \exp \left[-\int_{0}^{\beta} d \tau \sum_{\mu} \int d \mathbf{r} \bar{\phi}\left(\mathbf{r}, \mathbf{r}_{\mu}\right) \frac{1}{J \Omega} \phi\left(\mathbf{r}, \mathbf{r}_{\mu}\right)\right],
$$

with which a shift of the Bose field, $\phi\left(\mathbf{r}, \mathbf{r}_{\mu}\right) \rightarrow \phi\left(\mathbf{r}, \mathbf{r}_{\mu}\right)+J \Omega\left[\bar{\Psi}(\mathbf{r}, \tau) \tau_{A}^{T} \Psi\left(\mathbf{r}_{\mu}, \tau\right)+\bar{\Psi}\left(\mathbf{r}_{\mu}, \tau\right) \tau_{B}^{T} \Psi(\mathbf{r}, \tau)\right]$, yields

$$
\begin{aligned}
\mathcal{S}_{\text {pair }}= & \int_{0}^{\beta} d \tau d \mathbf{r} \bar{\Psi} \partial_{\tau} \Psi+\int_{0}^{\beta} d \tau d \mathbf{r} \bar{\Psi}(\mathbf{r}, \tau) H_{0}(\hat{\mathbf{k}}) \Psi(\mathbf{r}, \tau)+\sum_{\mu} \int_{0}^{\beta} d \tau d \mathbf{r} \bar{\phi}\left(\mathbf{r}, \mathbf{r}_{\mu}\right) \frac{1}{J \Omega} \phi\left(\mathbf{r}, \mathbf{r}_{\mu}\right) \\
& +\sum_{\mu} \int_{0}^{\beta} d \tau d \mathbf{r} \bar{\phi}\left(\mathbf{r}, \mathbf{r}_{\mu}\right)\left[\bar{\Psi}(\mathbf{r}) \tau_{A}^{T} \Psi\left(\mathbf{r}_{\mu}\right)+\bar{\Psi}\left(\mathbf{r}_{\mu}\right) \tau_{B}^{T} \Psi(\mathbf{r})\right] \\
& +\sum_{\mu} \int_{0}^{\beta} d \tau d \mathbf{r}\left[\bar{\Psi}\left(\mathbf{r}_{\mu}\right) \tau_{A} \Psi(\mathbf{r})+\bar{\Psi}(\mathbf{r}) \tau_{B} \Psi\left(\mathbf{r}_{\mu}\right)\right] \phi\left(\mathbf{r}, \mathbf{r}_{\mu}\right) .
\end{aligned}
$$

The fermion singlets, in the last two terms, can mediate an effective interaction between the order parameters $\phi\left(\mathbf{r}, \mathbf{r}_{\mu}\right)$ after being integrated out. To perform this integration, we go to the momentum-frequency space for the fermion Grassmann field,

$$
\Psi(\tau, \mathbf{r})=\frac{1}{\sqrt{\beta}} \frac{1}{\sqrt{S}} \sum_{\mathbf{k}} \sum_{\omega_{n}} \Psi\left(\omega_{n}, \mathbf{k}\right) e^{-i \omega_{n} \tau} e^{i \mathbf{k} \cdot \mathbf{r}},
$$

and for the boson field with respect to the center-of-mass coordinate $\mathbf{r}+\mathbf{r}_{\mu} / 2$,

$$
\phi\left(\tau, \mathbf{r}, \mathbf{r}+\mathbf{b}_{\mu}\right)=\frac{1}{\sqrt{\beta}} \frac{1}{\sqrt{S}} \sum_{\mathbf{k}, \omega_{m}} \phi\left(\omega_{m}, \mathbf{k}\right) e^{-i \omega_{n} \tau} e^{i \mathbf{k} \cdot\left(\mathbf{r}+\mathbf{b}_{\mu} / 2\right)} .
$$

With $k \equiv\left\{\omega_{n}, \mathbf{k}\right\}$ and $q \equiv\left\{\omega_{m}, \mathbf{q}\right\}$, we arrive at the partition function

$$
Z=\int \mathcal{D} \bar{\phi}_{\mu}(q) \mathcal{D} \phi_{\mu}(q) \exp \left(-\mathcal{S}_{\text {eff }}[\bar{\phi}, \phi]\right)
$$

with an effective action for the boson field,

$$
\mathcal{S}_{\text {eff }}[\bar{\phi}, \phi]=\sum_{\mu} \sum_{q} \bar{\phi}_{\mu}(q) \frac{1}{J \Omega} \phi_{\mu}(q)-\operatorname{tr} \ln \left[-\hat{G}^{-1}(\phi, \bar{\phi})\right] .
$$

Here, $\operatorname{tr}(\ldots)=\sum_{k}\langle k|\ldots| k\rangle, \hat{G}^{-1}[\phi]=i \hat{\omega}-\hat{H}_{0}-\hat{\Phi}$, in which the matrix elements of the operators read

$$
\begin{aligned}
\left\langle k|\hat{\Phi}| k^{\prime}\right\rangle & =\frac{1}{\sqrt{\beta S}} \sum_{\mu}\left[\bar{\phi}_{\mu}\left(k^{\prime}-k\right)\left(e^{i \boldsymbol{K} \cdot \mathbf{b}_{\mu}} \tau_{A}^{T}+e^{-i \boldsymbol{K} \cdot \mathbf{b}_{\mu}} \tau_{B}^{T}\right)+\phi_{\mu}\left(k-k^{\prime}\right)\left(e^{-i \boldsymbol{K} \cdot \mathbf{b}_{\mu}} \tau_{A}+e^{i \boldsymbol{K} \cdot \mathbf{b}_{\mu}} \tau_{B}\right)\right], \\
\left\langle k\left|\left(-i \hat{\omega}+\hat{H}_{0}\right)\right| k^{\prime}\right\rangle & =\left[-i \omega_{n}+\hat{H}_{0}(\mathbf{k})\right] \delta_{k k^{\prime}},
\end{aligned}
$$


with $\kappa=\left(\mathbf{k}+\mathbf{k}^{\prime}\right) / 2$ denoting the center-of-mass momentum. When the order parameter is small, it can be used to expand the action,

$$
\mathcal{S}_{\mathrm{eff}}[\bar{\phi}, \phi]=\sum_{\mu, q} \bar{\phi}_{\mu}(q) \frac{1}{J \Omega} \phi_{\mu}(q)+\frac{1}{2} \sum_{k k^{\prime}} \operatorname{tr}\left(G_{k}^{0} \Phi_{k, k^{\prime}} G_{k^{\prime}}^{0} \Phi_{k^{\prime}, k}\right)+\frac{1}{S} \int d \mathbf{r} \frac{1}{4} \sum_{k} \operatorname{tr}\left(G_{0 k} \Phi_{\mathbf{k}} G_{0 k} \Phi_{\mathbf{k}} G_{0 k} \Phi_{\mathbf{k}} G_{0 k} \Phi_{\mathbf{k}}\right),
$$

where the first two terms are linear, while the third term is nonlinear.

\section{Linear term}

From the first two terms in Eq. (A3), we arrive at the linear effective action,

$$
\mathcal{S}_{\text {eff }}^{L}=\sum_{\mu q} \bar{\phi}_{\mu}(q) \frac{1}{J \Omega} \phi_{\mu}(q)+\frac{1}{\beta S} \sum_{k q, \mu \mu^{\prime}} B_{k-\frac{q}{2}, k+\frac{q}{2}}^{\mu \mu^{\prime}} \bar{\phi}_{\mu}(q) \phi_{\mu^{\prime}}(q)
$$

where

$$
B_{k-\frac{q}{2}, k+\frac{q}{2}}^{\mu \mu^{\prime}}=\operatorname{tr}\left[G_{k-\frac{q}{2}}^{0}\left(e^{i \mathbf{k} \cdot \mathbf{b}_{\mu}} \tau_{A}^{T}+e^{-i \mathbf{k} \cdot \mathbf{b}_{\mu}} \tau_{B}^{T}\right) G_{k+\frac{q}{2}}^{0}\left(e^{-i \mathbf{k} \cdot \mathbf{b}_{\mu^{\prime}}} \tau_{A}+e^{i \mathbf{k} \cdot \mathbf{b}_{\mu^{\prime}}} \tau_{B}\right)\right] .
$$

Here, $G_{0}\left(\omega_{m}, \mathbf{k}\right)=\operatorname{diag}\left\{G_{e}\left(\omega_{m}, \mathbf{k}\right), G_{h}\left(\omega_{m}, \mathbf{k}\right)\right\}$ is the Green function of the free Hamiltonian in the Nambu space, where the Green functions in the electron and hole space read

$$
\begin{aligned}
& G_{e}\left(\mathbf{k}, \omega_{m}\right)=\frac{P_{1}(\mathbf{k})}{i \omega_{m}-\varepsilon_{1}(\mathbf{k})}+\frac{P_{2}(\mathbf{k})}{i \omega_{m}-\varepsilon_{2}(\mathbf{k})}, \\
& G_{h}\left(\mathbf{k}, \omega_{m}\right)=\frac{P_{1}(\mathbf{k})}{i \omega_{m}+\varepsilon_{1}(\mathbf{k})}+\frac{P_{2}(\mathbf{k})}{i \omega_{m}+\varepsilon_{2}(\mathbf{k})} .
\end{aligned}
$$

Here, $\varepsilon_{1}(\mathbf{k})=t|f(\mathbf{k})|-\mu$ and $\varepsilon_{2}(\mathbf{k})=-t|f(\mathbf{k})|-\mu$ are the electron and hole dispersions, and

$$
P_{1}(\mathbf{k})=\frac{1}{2}\left(\begin{array}{cc}
1 & -e^{i \phi_{\mathbf{k}}} \\
-e^{-i \phi_{\mathbf{k}}} & 1
\end{array}\right), \quad P_{2}(\mathbf{k})=\frac{1}{2}\left(\begin{array}{cc}
1 & e^{i \phi_{\mathbf{k}}} \\
e^{-i \phi_{\mathbf{k}}} & 1
\end{array}\right)
$$

are the projection operators for the two bands.

With Green function Eq. (A5), we obtain

$$
\begin{aligned}
& B_{k-\frac{q}{2}, k+\frac{q}{2}}^{\mu \mu^{\prime}}=\frac{1}{2}\left(\frac{1}{i \omega_{m}+\varepsilon_{1}\left(\mathbf{k}-\frac{\mathbf{q}}{2}\right)} \frac{1}{i \omega_{m}-\varepsilon_{1}\left(\mathbf{k}+\frac{\mathbf{q}}{2}\right)}+\frac{1}{i \omega_{m}+\varepsilon_{2}\left(\mathbf{k}-\frac{\mathbf{q}}{2}\right)} \frac{1}{i \omega_{m}-\varepsilon_{2}\left(\mathbf{k}+\frac{\mathbf{q}}{2}\right)}\right) \\
& \times\left\{\cos \left[\mathbf{k} \cdot\left(\mathbf{b}_{\mu}+\mathbf{b}_{\mu^{\prime}}\right)-\varphi_{\mathbf{k}-\frac{\mathrm{q}}{2}}-\varphi_{\mathbf{k}+\frac{\mathrm{q}}{2}}\right]+\cos \mathbf{k} \cdot\left(\mathbf{b}_{\mu}-\mathbf{b}_{\mu^{\prime}}\right)\right\} \\
& +\frac{1}{2}\left(\frac{1}{i \omega_{m}+\varepsilon_{1}\left(\mathbf{k}-\frac{\mathbf{q}}{2}\right)} \frac{1}{i \omega_{m}-\varepsilon_{2}\left(\mathbf{k}+\frac{\mathbf{q}}{2}\right)}+\frac{1}{i \omega_{m}+\varepsilon_{2}\left(\mathbf{k}-\frac{\mathbf{q}}{2}\right)} \frac{1}{i \omega_{m}-\varepsilon_{1}\left(\mathbf{k}+\frac{\mathbf{q}}{2}\right)}\right) \\
& \times\left\{-\cos \left[\mathbf{k} \cdot\left(\mathbf{b}_{\mu}+\mathbf{b}_{\mu^{\prime}}\right)-\varphi_{\mathbf{k}-\frac{\mathrm{q}}{2}}-\varphi_{\mathbf{k}+\frac{\mathrm{q}}{2}}\right]+\cos \mathbf{k} \cdot\left(\mathbf{b}_{\mu}-\mathbf{b}_{\mu^{\prime}}\right)\right\},
\end{aligned}
$$

which is an even function of $\mathbf{q}$. Assuming a small $\mathbf{q}$, we define the mass and fluctuation terms,

$$
\begin{aligned}
& \frac{1}{S} \frac{1}{\beta} \sum_{k} B_{k, k}^{\mu \mu^{\prime}}+\frac{1}{J \Omega} \delta_{\mu \mu^{\prime}} \equiv \mathcal{M}^{\mu \mu^{\prime}}(q=0), \\
& \left.\frac{1}{S} \frac{1}{\beta} \sum_{k}\left(B_{k-q / 2, k+q / 2}^{\mu \mu^{\prime}}-B_{k, k}^{\mu \mu^{\prime}}\right)\right|_{\omega_{q} \rightarrow 0, \mathbf{q} \rightarrow 0} \longrightarrow \sum_{\delta \gamma} \mathcal{T}_{\delta \gamma}^{\mu \mu^{\prime}} \mathbf{q}_{\delta} \mathbf{q}_{\gamma},
\end{aligned}
$$

where the fluctuation of long range is determined by

$$
\mathcal{T}_{\delta \gamma}^{\mu \mu^{\prime}} \equiv\left(\begin{array}{lll}
T^{11} & T^{12} & T^{13} \\
T^{12} & T^{22} & T^{23} \\
T^{13} & T^{23} & T^{33}
\end{array}\right)_{\delta \gamma}=\left.\frac{1}{2} \frac{\partial^{2}}{\partial \mathbf{q}_{\delta} \partial \mathbf{q}_{\gamma}}\left(\frac{1}{S} \frac{1}{\beta} \sum_{k} B_{k-\frac{q}{2}, k+\frac{q}{2}}^{\mu \mu^{\prime}}\right)\right|_{\omega_{q} \rightarrow 0, \mathbf{q} \rightarrow 0} .
$$

Via the summation over momentum and Matsubara frequency, we obtain

$$
\begin{aligned}
\mathcal{B}_{\mathbf{q}}^{\mu \mu^{\prime}} \equiv \frac{1}{S} \frac{1}{\beta} \sum_{k} B_{k-\frac{q}{2}, k+\frac{q}{2}}^{\mu \mu^{\prime}}= & \frac{1}{2 S} \sum_{\mathbf{k}}\left(\frac{n_{F}\left(\varepsilon_{1}\left(\mathbf{k}-\frac{\mathbf{q}}{2}\right)\right)+n_{F}\left(\varepsilon_{1}\left(\mathbf{k}+\frac{\mathbf{q}}{2}\right)\right)-1}{\varepsilon_{1}\left(\mathbf{k}-\frac{\mathbf{q}}{2}\right)+\varepsilon_{1}\left(\mathbf{k}+\frac{\mathbf{q}}{2}\right)}+\frac{n_{F}\left(\varepsilon_{2}\left(\mathbf{k}-\frac{\mathbf{q}}{2}\right)\right)+n_{F}\left(\varepsilon_{2}\left(\mathbf{k}+\frac{\mathbf{q}}{2}\right)\right)-1}{\varepsilon_{2}\left(\mathbf{k}-\frac{\mathbf{q}}{2}\right)+\varepsilon_{2}\left(\mathbf{k}+\frac{\mathbf{q}}{2}\right)}\right) \\
& \times\left\{\cos \left[\mathbf{k} \cdot\left(\mathbf{b}_{\mu}+\mathbf{b}_{\mu^{\prime}}\right)-\phi_{\mathbf{k}-\frac{\mathbf{q}}{2}}-\phi_{\mathbf{k}+\frac{\mathbf{q}}{2}}\right]+\cos \mathbf{k} \cdot\left(\mathbf{b}_{\mu}-\mathbf{b}_{\mu^{\prime}}\right)\right\}
\end{aligned}
$$




$$
\begin{aligned}
& +\frac{1}{2 S} \sum_{\mathbf{k}}\left(\frac{n_{F}\left(\varepsilon_{1}\left(\mathbf{k}-\frac{\mathbf{q}}{2}\right)\right)+n_{F}\left(\varepsilon_{2}\left(\mathbf{k}+\frac{\mathbf{q}}{2}\right)\right)-1}{\varepsilon_{1}\left(\mathbf{k}-\frac{\mathbf{q}}{2}\right)+\varepsilon_{2}\left(\mathbf{k}+\frac{\mathbf{q}}{2}\right)}+\frac{n_{F}\left(\varepsilon_{2}\left(\mathbf{k}-\frac{\mathbf{q}}{2}\right)\right)+n_{F}\left(\varepsilon_{1}\left(\mathbf{k}+\frac{\mathbf{q}}{2}\right)\right)-1}{\varepsilon_{2}\left(\mathbf{k}-\frac{\mathbf{q}}{2}\right)+\varepsilon_{1}\left(\mathbf{k}+\frac{\mathbf{q}}{2}\right)}\right) \\
& \times\left\{-\cos \left[\mathbf{k} \cdot\left(\mathbf{b}_{\mu}+\mathbf{b}_{\mu^{\prime}}\right)-\phi_{\mathbf{k}-\frac{\mathbf{q}}{2}}-\phi_{\mathbf{k}+\frac{\mathbf{q}}{2}}\right]+\cos \mathbf{k} \cdot\left(\mathbf{b}_{\mu}-\mathbf{b}_{\mu^{\prime}}\right)\right\} .
\end{aligned}
$$

Finally, the inverse Fourier transformation $\phi_{\mu}(q)=\frac{1}{\sqrt{S}} \frac{1}{\sqrt{\beta}} \int d \tau d \mathbf{r} e^{i \omega_{m} \tau} e^{-i \mathbf{q} \cdot\left(\mathbf{r}+\mathbf{b}_{\mu} / 2\right)} \phi_{\mu}(\tau, \mathbf{r})$ can bring the action back to the time-spatial space, yielding the effective action

$$
\begin{aligned}
\mathcal{S}_{\text {eff }}^{L}[\bar{\phi}, \phi]= & \sum_{\mu \mu^{\prime}} \int_{0}^{\beta} d \tau d \mathbf{r} \bar{\phi}_{\mu}(\mathbf{r}, \tau) \mathcal{M}^{\mu \mu^{\prime}} \phi_{\mu^{\prime}}(\mathbf{r}, \tau) \\
& +\sum_{\mu \mu^{\prime}} \sum_{\delta \gamma} \mathcal{T}_{\delta \gamma}^{\mu \mu^{\prime}} \int_{0}^{\beta} d \tau d \mathbf{r} \partial_{\delta} \phi_{\mu}^{*}(\mathbf{r}, \tau) \partial_{\gamma} \phi_{\mu^{\prime}}(\mathbf{r}, \tau) .
\end{aligned}
$$

The gap equation is given by the saddle-point solution $\delta S_{\text {eff }}(\bar{\phi}, \phi) /\left.\delta \bar{\phi}_{q}\right|_{q \rightarrow 0}=0$, leading to the eigenvalue equation for the vector fields $\vec{\Phi}=\left(\phi_{1}, \phi_{2}, \phi_{3}\right)^{T}$,

$$
\frac{1}{J \Omega} \vec{\Phi}=\left(\begin{array}{ccc}
\mathcal{A} & \mathcal{B} & \mathcal{B} \\
\mathcal{B} & \mathcal{A} & \mathcal{B} \\
\mathcal{B} & \mathcal{B} & \mathcal{A}
\end{array}\right) \vec{\Phi},
$$

where $\mathcal{A}=-\mathcal{B}_{\mathbf{q}=0}^{\mu \mu}$ and $\mathcal{B}=-\left.\mathcal{B}_{\mathbf{q}=0}^{\mu \mu^{\prime}}\right|_{\mu \neq \mu^{\prime}}$. The first eigenvalue $1 / J=\Omega(\mathcal{A}+2 \mathcal{B})$ corresponds to the extended $s$-wave state with eigenvector $\vec{\Delta}_{s}=(1,1,1)^{T} / \sqrt{3}$, and the remaining two eigenvalues $1 / J=\Omega(\mathcal{A}-\mathcal{B})$ are degenerate, corresponding to the $d$-wave order parameters with two degenerate eigenvectors,

$$
\vec{\Delta}_{a}=\frac{1}{\sqrt{6}}(2,-1,-1)^{T}, \quad d_{x^{2}-y^{2}}, \quad \vec{\Delta}_{b}=\frac{1}{\sqrt{2}}(0,1,-1)^{T}, \quad d_{x y} .
$$

The superposition of $\vec{\Delta}_{a}$ and $\vec{\Delta}_{b}$ gives different types of $d$-wave superconductivity. Particularly, superposition $\frac{\sqrt{2}}{2}\left(\vec{\Delta}_{a}+i \vec{\Delta}_{b}\right)=$ $\frac{1}{\sqrt{3}}\left(1, e^{i \frac{2 \pi}{3}}, e^{i \frac{4 \pi}{3}}\right)^{T}$ gives the $d_{x^{2}-y^{2}}+i d_{x y}$ wave, while $\frac{\sqrt{2}}{2}\left(\vec{\Delta}_{a}-i \vec{\Delta}_{b}\right)=\frac{1}{\sqrt{3}}\left(1, e^{i \frac{4 \pi}{3}}, e^{i \frac{2 \pi}{3}}\right)^{T}$ gives the $d_{x^{2}-y^{2}}-i d_{x y}$ wave. We decompose $\phi_{\mu}$ by the new basis with

$$
\Phi(\mathbf{r}, t)=\left[\phi_{1}(\mathbf{r}, t), \phi_{2}(\mathbf{r}, t), \phi_{3}(\mathbf{r}, t)\right]^{T}=\eta_{1}(\mathbf{r}, t) \xi_{1}+\eta_{2}(\mathbf{r}, t) \xi_{2}+\eta_{3}(\mathbf{r}, t) \xi_{3},
$$

where

$$
\begin{aligned}
& \xi_{1}=\frac{1}{\sqrt{3}}\left(1, e^{i 2 \pi / 3}, e^{i 4 \pi / 3}\right)^{T}, \\
& \xi_{2}=\frac{1}{\sqrt{3}}\left(1, e^{i 4 \pi / 3}, e^{i 2 \pi / 3}\right)^{T}, \\
& \xi_{3}=\frac{1}{\sqrt{3}}(1,1,1)^{T}
\end{aligned}
$$

are the basis for the order parameters in the $\left(d_{x^{2}-y^{2}}+i d_{x y}\right)$ wave, $\left(d_{x^{2}-y^{2}}-i d_{x y}\right)$ wave, and $s$ wave, respectively, and $\eta_{i}$ denote the amplitudes of these order parameters.

In terms of order-parameter amplitudes $\eta_{i}(\mathbf{r})$, we express the linear GL Lagrangian as

$$
\begin{aligned}
L_{\mathrm{eff}} & =\sum_{i=\{1,2,3\}}\left(\xi_{i}^{\dagger} \mathcal{M} \xi_{i}\right) \int d \mathbf{r} \eta_{i}^{*}(\mathbf{r}) \eta_{i}(\mathbf{r})+\sum_{\delta \gamma} \sum_{i j}\left(\xi_{i}^{\dagger} \mathcal{T}_{\delta \gamma} \xi_{j}\right) \int d \mathbf{r} \partial_{\delta} \eta_{i}^{*}(\mathbf{r}) \partial_{\gamma} \eta_{j}(\mathbf{r}) \\
& =\sum_{i} a_{i} \int d \mathbf{r} \eta_{i}^{*}(\mathbf{r}) \eta_{i}(\mathbf{r})+\sum_{\delta \gamma} \sum_{i j} c_{\delta \gamma}^{i j} \int d \mathbf{r} \partial_{\delta} \eta_{i}^{*} \partial_{\gamma} \eta_{j},
\end{aligned}
$$

where the coefficients $a_{i} \equiv \xi_{i}^{\dagger} \mathcal{M} \xi_{i}$ and $c_{\delta \gamma}^{i j} \equiv \xi_{i}^{\dagger} \mathcal{T}_{\delta \gamma} \xi_{j}$ are governed by symmetry and band structure. These coefficients are simply parameterized by several real quantities $\left\{a, a^{\prime}, b, b^{\prime}, e_{1}, e_{2}\right\}$. For $a_{i}, a_{1}=a_{2}=a$ and $a_{3}=a^{\prime}$, following the gap equation near $T_{c}$. When $i=j=\{1,2,3\}$,

$$
c_{\delta \gamma}^{11}=c_{\delta \gamma}^{22}=b \delta_{\delta \gamma}, \quad c_{\delta \gamma}^{33}=b^{\prime} \delta_{\delta \gamma}
$$

when $i \neq j$, we find

$$
c_{x x}^{21}=-c_{y y}^{21}=e_{1}(1-i \sqrt{3}), \quad c_{x y}^{21}=c_{y x}^{21}=i e_{1}(1-i \sqrt{3}),
$$




$$
\begin{aligned}
& c_{x x}^{31}=-c_{y y}^{31}=e_{2}(1+i \sqrt{3}), \quad c_{x y}^{31}=c_{y x}^{31}=-i e_{2}(1+i \sqrt{3}), \\
& c_{x x}^{32}=-c_{y y}^{32}=e_{2}(1-i \sqrt{3}), \quad c_{x y}^{32}=c_{y x}^{32}=i e_{2}(1-i \sqrt{3}),
\end{aligned}
$$

and $c_{\delta \gamma}^{j i}=\left(c_{\delta \gamma}^{i j}\right)^{*}$ that guarantees the Hermiticity. These analyses simplify the linear GL Lagrangian to be

$$
\begin{aligned}
L_{\mathrm{eff}}= & a \int d \mathbf{r}\left[\left|\eta_{1}(\mathbf{r})\right|^{2}+\left|\eta_{2}(\mathbf{r})\right|^{2}\right]+a^{\prime} \int d \mathbf{r}\left|\eta_{3}(\mathbf{r})\right|^{2} \\
+ & b \int d \mathbf{r}\left(\left|\partial_{x} \eta_{1}\right|^{2}+\left|\partial_{y} \eta_{1}\right|^{2}+\left|\partial_{x} \eta_{2}\right|^{2}+\left|\partial_{y} \eta_{2}\right|^{2}\right) \\
+ & b^{\prime} \int d \mathbf{r}\left[\left|\partial_{x} \eta_{3}(\mathbf{r})\right|^{2}+\left|\partial_{y} \eta_{3}(\mathbf{r})\right|^{2}\right] \\
+ & {\left[e_{1}(1-i \sqrt{3}) \int d \mathbf{r}\left(\partial_{x}+i \partial_{y}\right) \eta_{2}^{*}(\mathbf{r})\left(\partial_{x}+i \partial_{y}\right) \eta_{1}(\mathbf{r})\right.} \\
& \quad+e_{2}(1+i \sqrt{3}) \int d \mathbf{r}\left(\partial_{x}-i \partial_{y}\right) \eta_{3}^{*}(\mathbf{r})\left(\partial_{x}-i \partial_{y}\right) \eta_{1}(\mathbf{r}) \\
& \left.+e_{2}(1-i \sqrt{3}) \int d \mathbf{r}\left(\partial_{x}+i \partial_{y}\right) \eta_{3}^{*}(\mathbf{r})\left(\partial_{x}+i \partial_{y}\right) \eta_{2}(\mathbf{r})+\text { H.c. }\right],
\end{aligned}
$$

where the coefficients with different chemical potentials are numerically calculated in this work. The form of the gradient terms can be simply understood from the angular-momentum conservation. The system has $C_{6}$ symmetry, given by operators, e.g., $\hat{C}_{6}^{1}=e^{i \frac{i \pi}{6} J_{z}}$. Since $\partial_{x}+i \partial_{y}$ and $\partial_{x}-i \partial_{y}$ have angular momentum +1 and -1 , while $\eta_{1}, \eta_{2}$, and $\eta_{3}$ have angular momentum $+2,-2$, and 0 , the total angular momentum of every gradient term has to be the integer times of 6 . For example, the term $\int d \mathbf{r}\left(\partial_{x}+i \partial_{y}\right) \eta_{2}^{*}(\mathbf{r})\left(\partial_{x}+i \partial_{y}\right) \eta_{1}(\mathbf{r})$ has an angular momentum 6.

\section{Nonlinear terms}

For the derivation of the nonlinear term, we can treat the order parameter to be homogeneous by

$$
\phi_{\mu}(\mathbf{k}=0)=\frac{1}{\sqrt{S}} \int d \mathbf{r} \phi_{\mu}(\mathbf{r}) \approx \sqrt{S} \phi_{\mu}(\mathbf{r})
$$

With $\Phi_{\mathbf{k}}=\frac{1}{\sqrt{\beta}} \sum_{\mu}\left[\bar{\phi}_{\mu} Q_{\mu}^{T}(\mathbf{k})+\phi_{\mu} Q_{\mu}(-\mathbf{k})\right]$, where $Q_{\mu}^{T}(\mathbf{k})=e^{i \mathbf{k} \cdot \mathbf{b}_{\mu}} \tau_{A}^{T}+e^{-i \mathbf{k} \cdot \mathbf{b}_{\mu}} \tau_{B}^{T}$, the dominant nonlinear term in the Lagrangian reads

$$
\begin{aligned}
L_{\mathrm{eff}}^{\mathrm{NL}}= & \frac{1}{4 \beta^{2} S} \int d \mathbf{r} \sum_{\mu_{i}} \operatorname{tr}\left\{G_{0 k}\left[\bar{\phi}_{\mu_{1}} Q_{\mu_{1}}^{T}(\mathbf{k})+\phi_{\mu_{1}} Q_{\mu_{1}}(-\mathbf{k})\right] G_{0 k}\left[\bar{\phi}_{\mu_{2}} Q_{\mu_{2}}^{T}(\mathbf{k})+\phi_{\mu_{2}} Q_{\mu_{2}}(-\mathbf{k})\right] G_{0 k}\right. \\
& \left.\times\left[\bar{\phi}_{\mu_{3}} Q_{\mu_{3}}^{T}(\mathbf{k})+\phi_{\mu_{3}} Q_{\mu_{3}}(-\mathbf{k})\right] G_{0 k}\left[\bar{\phi}_{\mu_{4}} Q_{\mu_{4}}^{T}(\mathbf{k})+\phi_{\mu_{4}} Q_{\mu_{4}}(-\mathbf{k})\right]\right\} .
\end{aligned}
$$

Although with complicated form, it is simplified by using

$$
Q_{\mu_{i}}^{T}=\left(\begin{array}{cc}
0 & 0 \\
\square_{\mu_{i}}^{T} & 0
\end{array}\right), \quad Q_{\mu_{i}}=\left(\begin{array}{cc}
0 & \square_{\mu_{i}} \\
0 & 0
\end{array}\right),
$$

and the Green function in the subspace,

$$
G_{0 k} Q_{\mu_{i}}^{T}=\left(\begin{array}{cc}
0 & 0 \\
G_{k}^{h} \square_{\mu_{i}}^{T} & 0
\end{array}\right), \quad G_{0 k} Q_{\mu_{i}}=\left(\begin{array}{cc}
0 & G_{k}^{e} \square_{\mu_{i}} \\
0 & 0
\end{array}\right),
$$

since $G_{0 k} Q_{\mu_{i}}^{T} G_{0 k} Q_{\mu_{j}}^{T}=0$, via which the nonlinear term is reduced to

$$
L_{\text {eff }}^{\mathrm{NL}}=\frac{1}{S} \int d \mathbf{r} \frac{1}{2 \beta^{2}} \sum_{k} \sum_{\mu_{i}} \bar{\phi}_{\mu_{1}} \phi_{\mu_{2}} \bar{\phi}_{\mu_{3}} \phi_{\mu_{4}} \operatorname{tr}\left[G_{k}^{h} \square_{\mu_{1}}^{T}(\mathbf{k}) G_{k}^{e} \square_{\mu_{2}}(-\mathbf{k}) G_{k}^{h} \square_{\mu_{3}}^{T}(\mathbf{k}) G_{k}^{e} \square_{\mu_{4}}(-\mathbf{k})\right] .
$$


With relation $\square_{\mu}(-\mathbf{k})=\square_{\mu}^{T}(\mathbf{k})=\left(\begin{array}{cc}0 & e^{i \mathbf{k} \cdot \mathbf{b}_{\mu}} \\ e^{-i \mathbf{k} \cdot \mathbf{b}_{\mu}} & 0\end{array}\right)$ in mind, we obtain

$$
L_{\mathrm{eff}}^{\mathrm{NL}}=\frac{1}{S} \int d \mathbf{r} \frac{1}{2 \beta^{2}} \sum_{k} \sum_{\mu_{i}} \bar{\phi}_{\mu_{1}} \phi_{\mu_{2}} \bar{\phi}_{\mu_{3}} \phi_{\mu_{4}} \times \operatorname{tr}\left[G_{0 k} Q_{\mu_{1}}^{T}(\mathbf{k}) G_{0 k} Q_{\mu_{2}}(-\mathbf{k}) G_{0 k} Q_{\mu_{3}}^{T}(\mathbf{k}) G_{0 k} Q_{\mu_{4}}(-\mathbf{k})\right] .
$$

Focusing on the $d$ waves, we have $\sum_{\mu} \bar{\phi}_{\mu}(\mathbf{k}) \Lambda_{\mu}(\mathbf{k})=\eta_{1}^{*} \Lambda_{1}(\mathbf{k})+\eta_{2}^{*} \Lambda_{2}(\mathbf{k})$, with

$$
\begin{aligned}
& \Lambda_{1}(\mathbf{k})=\frac{1}{\sqrt{3}}\left(\begin{array}{cc}
0 & e^{i \mathbf{k} \cdot \mathbf{b}_{1}}+e^{i \frac{4 \pi}{3}} e^{i \mathbf{k} \cdot \mathbf{b}_{2}}+e^{i \frac{2 \pi}{3}} e^{i \mathbf{k} \cdot \mathbf{b}_{3}} \\
e^{-i \mathbf{k} \cdot \mathbf{b}_{1}}+e^{i \frac{4 \pi}{3}} e^{-i \mathbf{k} \cdot \mathbf{b}_{2}}+e^{i \frac{2 \pi}{3}} e^{-i \mathbf{k} \cdot \mathbf{b}_{3}} & 0
\end{array}\right), \\
& \Lambda_{2}(\mathbf{k})=\frac{1}{\sqrt{3}}\left(\begin{array}{cc}
0 & e^{i \mathbf{k} \cdot \mathbf{b}_{1}}+e^{i \frac{2 \pi}{3}} e^{i \mathbf{k} \cdot \mathbf{b}_{2}}+e^{i \frac{4 \pi}{3}} e^{i \mathbf{k} \cdot \mathbf{b}_{3}} \\
e^{-i \mathbf{k} \cdot \mathbf{b}_{1}}+e^{i \frac{2 \pi}{3}} e^{-i \mathbf{k} \cdot \mathbf{b}_{2}}+e^{i \frac{4 \pi}{3}} e^{-i \mathbf{k} \cdot \mathbf{b}_{3}} & 0
\end{array}\right) .
\end{aligned}
$$

We see $\Lambda_{2}^{*}(-\mathbf{k})=\Lambda_{1}(\mathbf{k})$ or $\Lambda_{1}^{*}(-\mathbf{k})=\Lambda_{2}(\mathbf{k})$. The nonlinear part in the Lagrangian then becomes

$$
\begin{aligned}
L_{\mathrm{eff}}^{\mathrm{NL}}= & \frac{1}{S} \int d \mathbf{r} \frac{1}{2 \beta^{2}} \sum_{k} \operatorname{tr}\left\{G_{k}^{h}\left[\eta_{1}^{*}(\mathbf{r}) \Lambda_{1}(\mathbf{k})+\eta_{2}^{*}(\mathbf{r}) \Lambda_{2}(\mathbf{k})\right] G_{k}^{e}\left[\eta_{1}(\mathbf{r}) \Lambda_{2}(\mathbf{k})+\eta_{2}(\mathbf{r}) \Lambda_{1}(\mathbf{k})\right]\right. \\
& \left.\times G_{k}^{h}\left[\eta_{1}^{*}(\mathbf{r}) \Lambda_{1}(\mathbf{k})+\eta_{2}^{*}(\mathbf{r}) \Lambda_{2}(\mathbf{k})\right] G_{k}^{e}\left[\eta_{1}(\mathbf{r}) \Lambda_{2}(\mathbf{k})+\eta_{2}(\mathbf{r}) \Lambda_{1}(\mathbf{k})\right]\right\} .
\end{aligned}
$$

There are still many terms that should be further simplified. We can generally demonstrate that only the terms with two $\Lambda_{1}(\mathbf{k})$ and $\Lambda_{2}(\mathbf{k})$ can survive among them by the $C_{3}$ symmetry of the system, dramatically simplifying the calculations. With using $C_{3}^{-1} \mathbf{k} \cdot \mathbf{b}_{\mu}=\mathbf{k} \cdot C_{3} \mathbf{b}_{\mu}$, we know that a $C_{3}$ operation gives a transformation $\left\{\mathbf{b}_{1}, \mathbf{b}_{2}, \mathbf{b}_{3}\right\} \rightarrow\left\{\mathbf{b}_{2}, \mathbf{b}_{3}, \mathbf{b}_{1}\right\}$. Such a transformation then leads to

$$
\Lambda_{1}(\mathbf{k}) \rightarrow e^{-i 2 \pi / 3} \Lambda_{1}(\mathbf{k}), \quad \Lambda_{2}(\mathbf{k}) \rightarrow e^{i 2 \pi / 3} \Lambda_{2}(\mathbf{k})
$$

This transformation gives $C \rightarrow e^{ \pm i 2 \pi / 3} C=0$ apart from the terms with two $\Lambda_{1}(\mathbf{k})$ and $\Lambda_{2}(\mathbf{k})$. We write down all these (six) terms and analyze their property in a general way:

$$
\begin{aligned}
& \text { (1) }=\frac{1}{2 \beta^{2}} \frac{1}{S} \sum_{k} \operatorname{tr}\left[G_{k}^{h} \Lambda_{1}(\mathbf{k}) G_{k}^{e} \Lambda_{2}(\mathbf{k}) G_{k}^{h} \Lambda_{1}(\mathbf{k}) G_{k}^{e} \Lambda_{2}(\mathbf{k})\right] \rightarrow\left|\eta_{1}(\mathbf{r})\right|^{4}, \\
& \text { (2) }=\frac{1}{2 \beta^{2}} \frac{1}{S} \sum_{k} \operatorname{tr}\left[G_{k}^{h} \Lambda_{2}(\mathbf{k}) G_{k}^{e} \Lambda_{1}(\mathbf{k}) G_{k}^{h} \Lambda_{2}(\mathbf{k}) G_{k}^{e} \Lambda_{1}(\mathbf{k})\right] \rightarrow\left|\eta_{2}(\mathbf{r})\right|^{4}, \\
& \text { (3) }=\frac{1}{2 \beta^{2}} \frac{1}{S} \sum_{k} \operatorname{tr}\left[G_{k}^{h} \Lambda_{1}(\mathbf{k}) G_{k}^{e} \Lambda_{1}(\mathbf{k}) G_{k}^{h} \Lambda_{2}(\mathbf{k}) G_{k}^{e} \Lambda_{2}(\mathbf{k})\right] \rightarrow\left|\eta_{1}(\mathbf{r})\right|^{2}\left|\eta_{2}(\mathbf{r})\right|^{2}, \\
& \text { (4) }=\frac{1}{2 \beta^{2}} \frac{1}{S} \sum_{k} \operatorname{tr}\left[G_{k}^{h} \Lambda_{2}(\mathbf{k}) G_{k}^{e} \Lambda_{2}(\mathbf{k}) G_{k}^{h} \Lambda_{1}(\mathbf{k}) G_{k}^{e} \Lambda_{1}(\mathbf{k})\right] \rightarrow\left|\eta_{1}(\mathbf{r})\right|^{2}\left|\eta_{2}(\mathbf{r})\right|^{2}, \\
& \text { (5) }=\frac{1}{2 \beta^{2}} \frac{1}{S} \sum_{k} \operatorname{tr}\left[G_{k}^{h} \Lambda_{1}(\mathbf{k}) G_{k}^{e} \Lambda_{2}(\mathbf{k}) G_{k}^{h} \Lambda_{2}(\mathbf{k}) G_{k}^{e} \Lambda_{1}(\mathbf{k})\right] \rightarrow\left|\eta_{1}(\mathbf{r})\right|^{2}\left|\eta_{2}(\mathbf{r})\right|^{2}, \\
& \text { (6) }=\frac{1}{2 \beta^{2}} \frac{1}{S} \sum_{k} \operatorname{tr}\left[G_{k}^{h} \Lambda_{2}(\mathbf{k}) G_{k}^{e} \Lambda_{1}(\mathbf{k}) G_{k}^{h} \Lambda_{1}(\mathbf{k}) G_{k}^{e} \Lambda_{2}(\mathbf{k})\right] \rightarrow\left|\eta_{1}(\mathbf{r})\right|^{2}\left|\eta_{2}(\mathbf{r})\right|^{2} .
\end{aligned}
$$

We demonstrate below that these terms can gather to two coefficients in terms of $f_{1}$ and $f_{2}$.

We can demonstrate that (1) = (2). We note that $G_{h}\left(\mathbf{k},-\omega_{m}\right)=-G_{e}\left(\mathbf{k}, \omega_{m}\right)$, leading to

$$
\begin{aligned}
2 & =\frac{1}{2 \beta^{2}} \frac{1}{S} \sum_{\mathbf{k}} \sum_{\omega_{m}} \operatorname{tr}\left[G_{h}\left(\mathbf{k},-\omega_{m}\right) \Lambda_{2}(\mathbf{k}) G_{e}\left(\mathbf{k},-\omega_{m}\right) \Lambda_{1}(\mathbf{k}) G_{h}\left(\mathbf{k},-\omega_{m}\right) \Lambda_{2}(\mathbf{k}) G_{e}\left(\mathbf{k},-\omega_{m}\right) \Lambda_{1}(\mathbf{k})\right] \\
& =\frac{1}{2 \beta^{2}} \frac{1}{S} \sum_{\mathbf{k}} \sum_{\omega_{m}} \operatorname{tr}\left[G_{e}\left(\mathbf{k}, \omega_{m}\right) \Lambda_{2}(\mathbf{k}) G_{h}\left(\mathbf{k}, \omega_{m}\right) \Lambda_{1}(\mathbf{k}) G_{e}\left(\mathbf{k}, \omega_{m}\right) \Lambda_{2}(\mathbf{k}) G_{h}\left(\mathbf{k}, \omega_{m}\right) \Lambda_{1}(\mathbf{k})\right] \\
& =\frac{1}{2 \beta^{2}} \frac{1}{S} \sum_{\mathbf{k}} \sum_{\omega_{m}} \operatorname{tr}\left[G_{h}\left(\mathbf{k}, \omega_{m}\right) \Lambda_{1}(\mathbf{k}) G_{e}\left(\mathbf{k}, \omega_{m}\right) \Lambda_{2}(\mathbf{k}) G_{h}\left(\mathbf{k}, \omega_{m}\right) \Lambda_{1}(\mathbf{k}) G_{e}\left(\mathbf{k}, \omega_{m}\right) \Lambda_{2}(\mathbf{k})\right]=\text { (1). }
\end{aligned}
$$


We can also show that the two terms are real: We note that the Green function also satisfies $G_{h}^{*}\left(-\mathbf{k}, \omega_{m}\right)=-G_{e}\left(\mathbf{k}, \omega_{m}\right)$ or $G_{e}^{*}\left(-\mathbf{k}, \omega_{m}\right)=-G_{h}\left(\mathbf{k}, \omega_{m}\right)$. By taking the conjugation and using $\mathbf{k} \rightarrow-\mathbf{k}$,

$$
\begin{aligned}
(2)^{*} & =\frac{1}{2 \beta^{2}} \frac{1}{S} \sum_{\mathbf{k}} \sum_{\omega_{m}} \operatorname{tr}\left[G_{h}^{*}\left(-\mathbf{k}, \omega_{m}\right) \Lambda_{2}^{*}(-\mathbf{k}) G_{e}^{*}\left(-\mathbf{k}, \omega_{m}\right) \Lambda_{1}^{*}(-\mathbf{k}) G_{h}^{*}\left(-\mathbf{k}, \omega_{m}\right) \Lambda_{2}^{*}(-\mathbf{k}) G_{e}^{*}\left(-\mathbf{k}, \omega_{m}\right) \Lambda_{1}^{*}(-\mathbf{k})\right] \\
& =\frac{1}{2 \beta^{2}} \frac{1}{S} \sum_{\mathbf{k}} \sum_{\omega_{m}} \operatorname{tr}\left[G_{e}\left(\mathbf{k}, \omega_{m}\right) \Lambda_{1}(\mathbf{k}) G_{h}\left(\mathbf{k}, \omega_{m}\right) \Lambda_{2}(\mathbf{k}) G_{e}\left(\mathbf{k}, \omega_{m}\right) \Lambda_{1}(\mathbf{k}) G_{h}\left(\mathbf{k}, \omega_{m}\right) \Lambda_{2}(\mathbf{k})\right] \\
& =\frac{1}{2 \beta^{2}} \frac{1}{S} \sum_{\mathbf{k}} \sum_{\omega_{m}} \operatorname{tr}\left[G_{h}\left(\mathbf{k}, \omega_{m}\right) \Lambda_{2}(\mathbf{k}) G_{e}\left(\mathbf{k}, \omega_{m}\right) \Lambda_{1}(\mathbf{k}) G_{h}\left(\mathbf{k}, \omega_{m}\right) \Lambda_{2}(\mathbf{k}) G_{e}\left(\mathbf{k}, \omega_{m}\right) \Lambda_{1}(\mathbf{k})\right]=\text { (2). }
\end{aligned}
$$

We define (1) = (2) $=f_{1}+f_{2}$. On the other hand, it is similar to demonstrate (3) = (4) and (5) = (6). We can also demonstrate that (3) and (5) are real, e.g.,

$$
\begin{aligned}
(5 * & =\frac{1}{2 \beta^{2}} \frac{1}{S} \sum_{\mathbf{k}} \sum_{\omega_{m}} \operatorname{tr}\left[G_{h}^{*}\left(-\mathbf{k}, \omega_{m}\right) \Lambda_{1}^{*}(-\mathbf{k}) G_{e}^{*}\left(-\mathbf{k}, \omega_{m}\right) \Lambda_{2}^{*}(-\mathbf{k}) G_{h}^{*}\left(-\mathbf{k}, \omega_{m}\right) \Lambda_{2}^{*}(-\mathbf{k}) G_{e}^{*}\left(-\mathbf{k}, \omega_{m}\right) \Lambda_{1}^{*}(-\mathbf{k})\right] \\
& =\frac{1}{2 \beta^{2}} \frac{1}{S} \sum_{\mathbf{k}} \sum_{\omega_{m}} \operatorname{tr}\left[G_{e}\left(\mathbf{k}, \omega_{m}\right) \Lambda_{2}(\mathbf{k}) G_{h}\left(\mathbf{k}, \omega_{m}\right) \Lambda_{1}(\mathbf{k}) G_{e}\left(\mathbf{k}, \omega_{m}\right) \Lambda_{1}(\mathbf{k}) G_{h}\left(\mathbf{k}, \omega_{m}\right) \Lambda_{2}(\mathbf{k})\right] \\
& =\frac{1}{2 \beta^{2}} \frac{1}{S} \sum_{\mathbf{k}} \sum_{\omega_{m}} \operatorname{tr}\left[G_{e}\left(\mathbf{k},-\omega_{m}\right) \Lambda_{1}(\mathbf{k}) G_{h}\left(\mathbf{k},-\omega_{m}\right) \Lambda_{2}(\mathbf{k}) G_{e}\left(\mathbf{k},-\omega_{m}\right) \Lambda_{2}(\mathbf{k}) G_{h}\left(\mathbf{k},-\omega_{m}\right) \Lambda_{1}(\mathbf{k})\right] \\
& =\frac{1}{2 \beta^{2}} \frac{1}{S} \sum_{\mathbf{k}} \sum_{\omega_{m}} \operatorname{tr}\left[G_{h}\left(\mathbf{k}, \omega_{m}\right) \Lambda_{1}(\mathbf{k}) G_{e}\left(\mathbf{k}, \omega_{m}\right) \Lambda_{2}(\mathbf{k}) G_{h}\left(\mathbf{k}, \omega_{m}\right) \Lambda_{2}(\mathbf{k}) G_{e}\left(\mathbf{k}, \omega_{m}\right) \Lambda_{1}(\mathbf{k})\right]=\text { (5). }
\end{aligned}
$$

When we write (3) $+(5)=f_{1}-f_{2}$, the nonlinear part of the GL Lagrangian reads

$$
L_{\mathrm{eff}}^{\mathrm{NL}}=\int d \mathbf{r}\left[f_{1}\left(\left|\eta_{1}\right|^{2}+\left|\eta_{2}\right|^{2}\right)^{2}+f_{2}\left(\left|\eta_{1}\right|^{2}-\left|\eta_{2}\right|^{2}\right)^{2}\right] .
$$

We now can work out the concrete value of $f_{1}$ and $f_{2}$. We have

$$
\begin{aligned}
& f_{1}+f_{2}=\frac{1}{2 \beta^{2}} \frac{1}{S} \sum_{k} \operatorname{tr}\left[G_{k}^{h} \Lambda_{1}(\mathbf{k}) G_{k}^{e} \Lambda_{2}(\mathbf{k}) G_{k}^{h} \Lambda_{1}(\mathbf{k}) G_{k}^{e} \Lambda_{2}(\mathbf{k})\right] \\
& f_{1}-f_{2}=\frac{1}{2 \beta^{2}} \frac{1}{S} \sum_{k} \operatorname{tr}\left[G_{k}^{h} \Lambda_{1}(\mathbf{k}) G_{k}^{e} \Lambda_{1}(\mathbf{k}) G_{k}^{h} \Lambda_{2}(\mathbf{k}) G_{k}^{e} \Lambda_{2}(\mathbf{k})\right]+\frac{1}{2 \beta^{2}} \frac{1}{S} \sum_{k} \operatorname{tr}\left[G_{k}^{h} \Lambda_{1}(\mathbf{k}) G_{k}^{e} \Lambda_{2}(\mathbf{k}) G_{k}^{h} \Lambda_{2}(\mathbf{k}) G_{k}^{e} \Lambda_{1}(\mathbf{k})\right] .
\end{aligned}
$$

We restrict to a high electron doping, in which case the electron and hole Green functions are approximated by $G_{k}^{h} \approx \frac{P_{1}(\mathbf{k})}{i \omega_{m}+\varepsilon_{1}(\mathbf{k})}$ and $G_{k}^{e} \approx \frac{P_{1}(\mathbf{k})}{i \omega_{m}-\varepsilon_{1}(\mathbf{k})}$, with which, e.g.,

$$
f_{1}+f_{2} \simeq \frac{1}{2 \beta^{2}} \frac{1}{S} \sum_{\mathbf{k}} \sum_{\omega_{m}} \operatorname{tr}\left\{\frac{P_{1}(\mathbf{k}) \Lambda_{1}(\mathbf{k}) P_{1}(\mathbf{k}) \Lambda_{2}(\mathbf{k}) P_{1}(\mathbf{k}) \Lambda_{1}(\mathbf{k}) P_{1}(\mathbf{k}) \Lambda_{2}(\mathbf{k})}{\left[i \omega_{m}+\varepsilon_{1}(\mathbf{k})\right]^{2}\left[i \omega_{m}-\varepsilon_{1}(\mathbf{k})\right]^{2}}\right\} .
$$

We calculate the integral according to the residue theorem and arrive at

$$
\begin{aligned}
f_{1}+f_{2}= & \frac{1}{2 \beta} \frac{1}{S} \sum_{\mathbf{k}} \operatorname{tr}\left[P_{1}(\mathbf{k}) \Lambda_{1}(\mathbf{k}) P_{1}(\mathbf{k}) \Lambda_{2}(\mathbf{k}) P_{1}(\mathbf{k}) \Lambda_{1}(\mathbf{k}) P_{1}(\mathbf{k}) \Lambda_{2}(\mathbf{k})\right] \\
& \times\left(\frac{1}{4 \varepsilon_{1}^{2}(\mathbf{k})}\left\{n_{F}^{\prime}\left[\varepsilon_{1}(\mathbf{k})\right]+n_{F}^{\prime}\left[-\varepsilon_{1}(\mathbf{k})\right]\right\}+\frac{1}{4 \varepsilon_{1}^{3}(\mathbf{k})}\left\{-n_{F}\left[\varepsilon_{1}(\mathbf{k})\right]+n_{F}\left[-\varepsilon_{1}(\mathbf{k})\right]\right\}\right),
\end{aligned}
$$

where $n_{F}(z)=1 /\left(e^{\beta z}+1\right)$. A similar expression is obtained for $f_{1}-f_{2}$.

\section{APPENDIX B: CHIRAL EQUILIBRIUM STATE}

Here we use the GL Lagrangian to analyze the equilibrium state. We estimate the parameters by using $t=2.7 \mathrm{eV}, J=0.25 t$, and $|\mathbf{b}|=2.46 / \sqrt{3} \AA$ for the single-layer graphene. For the chemical potentials $\mu / t=\{0.85,0.9,0.95,1,1.05,1.1\}$, the critical temperature is calculated to be $T_{c}=\{1.23,4.74,19.18,40.05,15.98,2.95\} \mathrm{K}$, exhibiting a dome around the doping with $\mu=t$. Above or below this region, the superconductivity no longer exists. Increasing $J$ may exponentially increase $T_{c}$. We note that $e_{1}$ approaches zero when $\mu \rightarrow t$ and becomes opposite with $\mu>t$ and $\mu<t$. We then choose $T=1.5 \mathrm{~K}$ and dopings with $\mu / t=\{0.9,1.1\}$ as the typical cases to illustrate the chirality switch dynamics. 

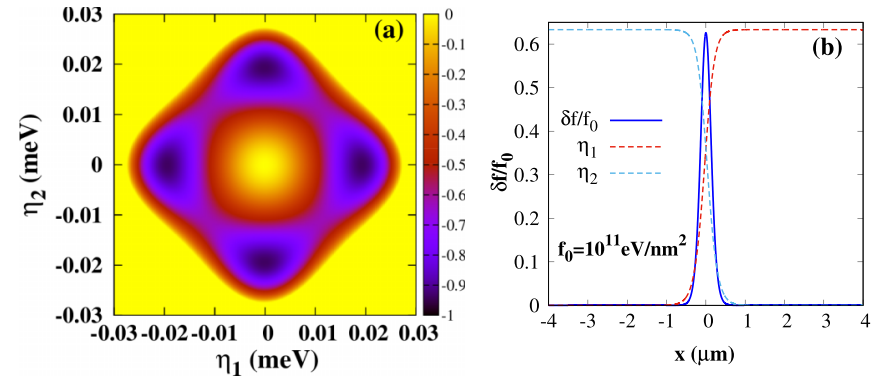

FIG. 5. Free-energy density of the chiral superconductor. In (a), the free-energy density with respect to the different values of $\eta_{1}$ and $\eta_{2}$ is plotted, in which the minimum represents the ground state of the superconductor. In (b), we plot the additional free energy $\delta f$ of the chirality domain wall in the one-dimensional case.

Without the external field, we may expect that the order parameter is homogeneous to minimize the free-energy density. We can demonstrate that $\left\{\left|\eta_{1}\right|,\left|\eta_{2}\right|\right\}=\eta_{0}\{1,0\}$ and $\eta_{0}\{0,1\}$ are two degenerate states that minimize the free-energy density. To this end,

$$
\begin{aligned}
\frac{\partial \mathcal{L}_{\text {eff }}}{\partial\left|\eta_{1}\right|}= & 2 a\left|\eta_{1}\right|+4 f_{1}\left(\left|\eta_{1}\right|^{2}+\left|\eta_{2}\right|^{2}\right)\left|\eta_{1}\right| \\
& +4 f_{2}\left(\left|\eta_{1}\right|^{2}-\left|\eta_{2}\right|^{2}\right)\left|\eta_{1}\right|=0, \\
\frac{\partial \mathcal{L}_{\text {eff }}}{\partial\left|\eta_{2}\right|}= & 2 a\left|\eta_{2}\right|+4 f_{1}\left(\left|\eta_{1}\right|^{2}+\left|\eta_{2}\right|^{2}\right)\left|\eta_{2}\right| \\
& -4 f_{2}\left(\left|\eta_{1}\right|^{2}-\left|\eta_{2}\right|^{2}\right)\left|\eta_{2}\right|=0 .
\end{aligned}
$$

The solutions give that the local minima are degenerate:

$$
\begin{aligned}
& \left|\eta_{1}\right|=0, \quad\left|\eta_{2}\right|=\sqrt{-\frac{a}{2\left(f_{1}+f_{2}\right)}} \equiv \eta_{0}, \\
& \left|\eta_{2}\right|=0, \quad\left|\eta_{1}\right|=\sqrt{-\frac{a}{2\left(f_{1}+f_{2}\right)}} .
\end{aligned}
$$

The superconductivity is spontaneously broken into one of the two classes. In Fig. 5(a), with parameters of $\mu=0.9 t$, the profile of the free energy is plotted with respect to $\left|\eta_{1}\right|$ and $\left|\eta_{2}\right|$, in which we calculate $\eta_{0}=0.02 \mathrm{meV}$ that identifies the minimum of free energy. The free energy is normalized by $f_{0}=10^{11} \mathrm{eV} / \mathrm{nm}^{2}$. In Fig. 5(b), we calculate and plot the additional free energy $\delta f$ of the chirality domain wall in the one-dimensional case. We see that the surface energy of the domain wall is positive and hence the domain is metastable. With our parameters, the wall width is about $1 \mu \mathrm{m}$.

On the basis of the chiral domain in Figs. 4(b) and 4(c), we now apply similar optical pulses, but use the left-circularly polarized laser that favors the switching from $\eta_{2}$ to $\eta_{1}$. In Fig. 6, we show the erasure of the chiral domain. The diffusion of order parameters at the edge leaves a ring of chiral domain with a small size, which, however, can be erased by pulses of larger size, such as $\sigma_{r}=90 \mu \mathrm{m}$, shown in Fig. 6(b).

\section{APPENDIX C: IMPLEMENTING HADAMARD GATES USING MAJORANA EDGE MODES}

The ability to optically control the topography of chiral superconducting domains provides a handle to implement



FIG. 6. Optical erasure of a chiral domain (normalized by $\eta_{0}$ ) in real space by laser spots of different sizes: (a1)-(a4) $\sigma_{r}=60 \mu \mathrm{m}$ and (b1)-(b4) $\sigma_{r}=90 \mu \mathrm{m}$. The spot sizes $\sqrt{2} \sigma_{r}$ are indicated by black circles. The second pulse is left-circularly polarized and favors the switching from $\eta_{2}$ to $\eta_{1}$, as shown by the snapshots (a4) and (b4).

quantum operations via interference of Majorana modes. Here, the key idea is a straightforward extension of a recent proposal [4] that exploits the domain-wall modes at interfaces between $p+i p$ superconductors and quantum anomalous Hall insulators, whose edges carry single Majorana and single complex fermions (which can equivalently be represented as pairs of Majorana fermions), respectively. To translate this idea to optically controlled $p+i p$ chiral superconductors, note that domain walls between $p+i p$ and $p-i p$ chiral domains similarly carry two Majorana modes, and hence act in analogy to the edge states of a quantum anomalous Hall insulator, depicted in Fig. 1 in the main text.

In this scheme, presume that two metallic leads are patterned over two domain walls between $p+i p / p-i p$ domains, as shown in Fig. 1 of the main text. The single-electron charging states $|0\rangle,|1\rangle$ of the two leads now define two singlequbit states: as one injects single electrons into the leads, these propagate chirally along the domain-wall boundary until they reach the sample edge (or, equivalently, an interface with a trivial insulator). At this point, the complex fermion edge mode fractionalizes into two Majorana modes, which entails a fractionalization of the injected electron. As each Majorana fermion proceeds to travel along the boundary mode and completes a revolution around the circuit, one can see that for the left-side circuit in Fig. 1, two of the Majorana fermions that originate from an electron injected into the left-side and right-side lead are exchanged. If the left-side and right-side charging states are represented via Majorana operators,

$$
\begin{aligned}
& \hat{c}_{L}: \hat{\gamma}_{L}^{1}, \hat{\gamma}_{L}^{2}, \\
& \hat{c}_{R}: \hat{\gamma}_{R}^{1}, \hat{\gamma}_{R}^{2},
\end{aligned}
$$

respectively, the resulting operation follows from an exchange,

$$
\begin{aligned}
& \hat{\gamma}_{L}^{2} \rightarrow \hat{\gamma}_{R}^{2}, \\
& \hat{\gamma}_{R}^{2} \rightarrow-\hat{\gamma}_{L}^{2} .
\end{aligned}
$$

Expressed in a charging basis, this exchange implements a non-Abelian operation. For instance, if the initial charging 
states take the form $|1\rangle|0\rangle$, then the operation transforms this into

$$
|1\rangle|0\rangle \rightarrow \frac{1}{\sqrt{2}}(|1\rangle|0\rangle+|0\rangle|1\rangle),
$$

thereby implementing the desired Hadamard gate.
Crucially, the order of the above operations is determined solely via the topography of the domain walls. For instance, a suitable change to the topography in Fig. 1 (right) changes the Hadamard gate to an identity operation. This permits control of the respective quantum operations via optical means.
[1] N. Read and D. Green, Paired states of fermions in two dimensions with breaking of parity and time-reversal symmetries and the fractional quantum Hall effect, Phys. Rev. B 61, 10267 (2000).

[2] S. R. Elliott and M. Franz, Colloquium: Majorana fermions in nuclear, particle, and solid-state physics, Rev. Mod. Phys. 87, 137 (2015).

[3] D. Aasen, M. Hell, R. V. Mishmash, A. Higginbotham, J. Danon, M. Leijnse, T. S. Jespersen, J. A. Folk, C. M. Marcus, K. Flensberg, and J. Alicea, Milestones Toward Majorana-Based Quantum Computing, Phys. Rev. X 6, 031016 (2016).

[4] B. Lian, X.-Q. Sun, A. Vaezi, X.-L. Qi, and S.-C. Zhang, Topological quantum computation based on chiral Majorana fermions, Proc. Natl. Acad. Sci. USA 115, 10938 (2018).

[5] S. D. Bader and S. S. P. Parkin, Spintronics, Annu. Rev. Condens. Matter Phys. 1, 71 (2010).

[6] I. Žutić, J. Fabian, and S. Das Sarma, Spintronics: Fundamentals and applications, Rev. Mod. Phys. 76, 323 (2004).

[7] J. R. Schaibley, H. Yu, G. Clark, P. Rivera, J. S. Ross, K. L. Seyler, W. Yao, and $\mathrm{X}$. Xu, Valleytronics in 2D materials, Nat. Rev. Mater. 1, 16055 (2016).

[8] G. Wang, A. Chernikov, M. M. Glazov, T. F. Heinz, X. Marie, T. Amand, and B. Urbaszek, Colloquium: Excitons in atomically thin transition metal dichalcogenides, Rev. Mod. Phys. 90, 021001 (2018).

[9] Z.-L. Xiang, S. Ashhab, J. Q. You, and F. Nori, Hybrid quantum circuits: Superconducting circuits interacting with other quantum systems, Rev. Mod. Phys. 85, 623 (2013).

[10] M. Claassen, D. M. Kennes, M. Zingl, M. A. Sentef, and A. Rubio, Universal optical control of chiral superconductors and Majorana modes, Nat. Phys. 15, 766 (2019).

[11] M. Bukov, L. D’Alessio, and A. Polkovnikov, Universal high-frequency behavior of periodically driven systems: From dynamical stabilization to Floquet engineering, Adv. Phys. 64, 139 (2015).

[12] A. Eckardt, Colloquium: Atomic quantum gases in periodically driven optical lattices, Rev. Mod. Phys. 89, 011004 (2017).

[13] C. Vaswani, M. Mootz, C. Sundahl, D. H. Mudiyanselage, J. H. Kang, X. Yang, D. Cheng, C. Huang, R. H. J. Kim, Z. Liu, L. Luo, I. E. Perakis, C. B. Eom, and J. Wang, Terahertz Second-Harmonic Generation from Lightwave Acceleration of Symmetry-Breaking Nonlinear Supercurrents, Phys. Rev. Lett. 124, 207003 (2020).

[14] S. Nakamura, K. Katsumi, H. Terai, and R. Shimano, Nonreciprocal Terahertz Second-Harmonic Generation in Superconducting NbN under Supercurrent Injection, Phys. Rev. Lett. 125, 097004 (2020).

[15] N. Kopnin, Theory of Nonequilibrium Superconductivity (Oxford University Press, New York, 2001).
[16] L. P. Gor'kov, Microscopic derivation of the Ginzburg-Landau equations in the theory of superconductivity, Sov. Phys. JETP 9, 1364 (1959).

[17] R. Nandkishore, L. S. Levitov, and A. V. Chubukov, Chiral superconductivity from repulsive interactions in doped graphene, Nat. Phys. 8, 158 (2012).

[18] A. M. Black-Schaffer and C. Honerkamp, Chiral $d$-wave superconductivity in doped graphene, J. Phys.: Condens. Matter 26, 423201 (2014).

[19] D. Vollhardt, Normal ${ }^{3} \mathrm{He}$ : An almost localized Fermi liquid, Rev. Mod. Phys. 56, 99 (1984).

[20] F. C. Zhang, C. Gros, T. M. Rice, and H. Shiba, A renormalised Hamiltonian approach to a resonant valence bond wavefunction, Supercond. Sci. Technol. 1, 36 (1988).

[21] M. Sigrist and K. Ueda, Phenomenological theory of unconventional superconductivity, Rev. Mod. Phys. 63, 239 (1991).

[22] P. Rosenzweig, H. Karakachian, S. Link, K. Küster, and U. Starke, Tuning the doping level of graphene in the vicinity of the Van Hove singularity via ytterbium intercalation, Phys. Rev. B 100, 035445 (2019).

[23] P. Rosenzweig, H. Karakachian, D. Marchenko, K. Küster, and U. Starke, Overdoping Graphene beyond the Van Hove Singularity, Phys. Rev. Lett. 125, 176403 (2020).

[24] Y. Cao, V. Fatemi, S. Fang, K. Watanabe, T. Taniguchi, E. Kaxiras, and P. Jarillo-Herrero, Unconventional superconductivity in magic-angle graphene superlattices, Nature (London) 556, 43 (2018).

[25] C. K. Xu and L. Balents, Topological Superconductivity in Twisted Multilayer Graphene, Phys. Rev. Lett. 121, 087001 (2018).

[26] D. M. Kennes, J. Lischner, and C. Karrasch, Strong correlations and $d+i d$ superconductivity in twisted bilayer graphene, Phys. Rev. B 98, 241407(R) (2018).

[27] L. Xian, D. M. Kennes, N. Tancogne-Dejean, M. Altarelli, and A. Rubio, Multiflat bands and strong correlations in twisted bilayer boron nitride: Doping-induced correlated insulator and superconductor, Nano Lett. 19, 4934 (2019).

[28] M. Yankowitz, S. Chen, H. Polshyn, Y. Zhang, K. Watanabe, T. Taniguchi, D. Graf, A. F. Young, and C. R. Dean, Tuning superconductivity in twisted bilayer graphene, Science 363, 1059 (2019).

[29] X. Lu, P. Stepanov, W. Yang, M. Xie, M. A. Aamir, I. Das, C. Urgell, K. Watanabe, T. Taniguchi, G. Zhang et al., Superconductors, orbital magnets and correlated states in magic-angle bilayer graphene, Nature (London) 574, 653 (2019).

[30] L. Wang, E.-M. Shih, A. Ghiotto, L. Xian, D. A. Rhodes, C. Tan, M. Claassen, D. M. Kennes, Y. Bai, B. Kim et al., Correlated electronic phases in twisted bilayer transition metal dichalcogenides, Nat. Mater. 19, 861 (2020). 
[31] A. Fischer, L. Klebl, C. Honerkamp, and D. M. Kennes, Spin-fluctuation-induced pairing in twisted bilayer graphene, Phys. Rev. B 103, L041103 (2021).

[32] J. Y. Lee, E. Khalaf, S. Liu, X. Liu, Z. Hao, P. Kim, and A. Vishwanath, Theory of correlated insulating behavior and spintriplet superconductivity in twisted double bilayer graphene, Nat. Commun. 10, 5333 (2019).

[33] G. M. Luke, A. Keren, L. P. Le, W. D. Wu, Y. J. Uemura, D. A. Bonn, L. Taillefer, and J. D. Garrett, Muon Spin Relaxation in $\mathrm{UPt}_{3}$, Phys. Rev. Lett. 71, 1466 (1993).

[34] R. Joynt and L. Taillefer, The superconducting phases of $\mathrm{UPt}_{3}$, Rev. Mod. Phys. 74, 235 (2002).

[35] K. E. Avers, W. J. Gannon, S. J. Kuhn, W. P. Halperin, J. A. Sauls, L. DeBeer-Schmitt, C. D. Dewhurst, J. Gavilano, G. Nagy, U. Gasser, and M. R. Eskildsen, Broken time-reversal symmetry in the topological superconductor $\mathrm{UPt}_{3}$, Nat. Phys. 16, 531 (2020).

[36] L. Jiao, S. Howard, S. Ran, Z. Y. Wang, J. O. Rodriguez, M. Sigrist, Z. Q. Wang, N. P. Butch, and V. Madhavan, Chiral superconductivity in heavy-fermion metal $\mathrm{UTe}_{2}$, Nature (London) 579, 523 (2020).

[37] G. M. Luke, Y. Fudamoto, K. M. Kojima, M. I. Larkin, J. Merrin, B. Nachumi, Y. J. Uemura, Y. Maeno, Z. Q. Mao, Y. Mori, H. Nakamura, and M. Sigrist, Time-reversal symmetrybreaking superconductivity in $\mathrm{Sr}_{2} \mathrm{RuO}_{4}$, Nature (London) 394, 558 (1998).

[38] A. P. Mackenzie and Y. Maeno, The superconductivity of $\mathrm{Sr}_{2} \mathrm{RuO}_{4}$ and the physics of spin-triplet pairing, Rev. Mod. Phys. 75, 657 (2003).

[39] A. Pustogow, Y. Luo, A. Chronister, Y. S. Su, D. A. Sokolov, F. Jerzembeck, A. P. Mackenzie, C. W. Hicks, N. Kikugawa, S. Raghu, E. D. Bauer, and S. E. Brown, Constraints on the superconducting order parameter in $\mathrm{Sr}_{2} \mathrm{RuO}_{4}$ from oxygen17 nuclear magnetic resonance, Nature (London) 574, 72 (2019).

[40] S. Benhabib, C. Lupien, I. Paul, L. Berges, M. Dion, M. Nardone, A. Zitouni, Z. Q. Mao, Y. Maeno, A. Georges, L. Taillefer, and C. Proust, Ultrasound evidence for a twocomponent superconducting order parameter in $\mathrm{Sr}_{2} \mathrm{RuO}_{4}$, Nat. Phys. 17, 194 (2021).

[41] S. Ghosh, A. Shekhter, F. Jerzembeck, N. Kikugawa, D. A. Sokolov, M. Brando, A. P. Mackenzie, C. W. Hicks, and B. J. Ramshaw, Thermodynamic evidence for a two-component superconducting order parameter in $\mathrm{Sr}_{2} \mathrm{RuO}_{4}$, Nat. Phys. 17, 199 (2021).

[42] S. A. Kivelson, A. C. Yuan, B. Ramshaw, and R. Thomale, A proposal for reconciling diverse experiments on the superconducting state in $\mathrm{Sr}_{2} \mathrm{RuO}_{4}$, npj Quantum Mater. 5, 43 (2020).

[43] Y. Nambu, Quasi-particles and gauge invariance in the theory of superconductivity, Phys. Rev. 117, 648 (1960).

[44] R. Matsunaga, N. Tsuji, H. Fujita, A. Sugioka, K. Makise, Y. Uzawa, H. Terai, Z. Wang, H. Aoki, and R. Shimano, Lightinduced collective pseudospin precession resonating with Higgs mode in a superconductor, Science 345, 1145 (2014).

[45] R. Matsunaga, N. Tsuji, K. Makise, H. Terai, H. Aoki, and R. Shimano, Polarization-resolved terahertz third-harmonic generation in a single-crystal superconductor $\mathrm{NbN}$ : Dominance of the Higgs mode beyond the BCS approximation, Phys. Rev. B 96, 020505(R) (2017).
[46] T. Cea, C. Castellani, and L. Benfatto, Nonlinear optical effects and third-harmonic generation in superconductors: Cooper pairs versus Higgs mode contribution, Phys. Rev. B 93, 180507(R) (2016).

[47] K. Katsumi, N. Tsuji, Y. I. Hamada, R. Matsunaga, J Schneeloch, R. D. Zhong, G. D. Gu, H. Aoki, Y. Gallais, and R. Shimano, Higgs Mode in the $d$-Wave Superconductor $\mathrm{Bi}_{2} \mathrm{Sr}_{2} \mathrm{CaCu}_{2} \mathrm{O}_{8+x}$ Driven by an Intense Terahertz Pulse, Phys. Rev. Lett. 120, 117001 (2018).

[48] N. Tsuji and H. Aoki, Theory of Anderson pseudospin resonance with Higgs mode in superconductors, Phys. Rev. B 92 064508 (2015).

[49] A. F. Kemper, M. A. Sentef, B. Moritz, J. K. Freericks, and T. P. Devereaux, Direct observation of Higgs mode oscillations in the pump-probe photoemission spectra of electron-phonon mediated superconductors, Phys. Rev. B 92, 224517 (2015).

[50] T. Yu and M. W. Wu, Gauge-invariant theory of quasiparticle and condensate dynamics in response to terahertz optical pulses in superconducting semiconductor quantum wells. I. $s$ wave superconductivity in the weak spin-orbit coupling limit, Phys. Rev. B 96, 155311 (2017).

[51] J. S. Neergaard-Nielsen, M. Takeuchi, K. Wakui, H. Takahashi, K. Hayasaka, M. Takeoka, and M. Sasaki, Optical ContinuousVariable Qubit, Phys. Rev. Lett. 105, 053602 (2010).

[52] J. Wenger, R. Tualle-Brouri, and P. Grangier, Non-Gaussian Statistics from Individual Pulses of Squeezed Light, Phys. Rev. Lett. 92, 153601 (2004).

[53] J. S. Neergaard-Nielsen, B. M. Nielsen, C. Hettich, K. Molmer, and E. S. Polzik, Generation of a Superposition of Odd Photon Number States for Quantum Information Networks, Phys. Rev. Lett. 97, 083604 (2006).

[54] A. Altland and B. Simons, Condensed Matter Field Theory (Cambridge University Press, Cambridge, 2010).

[55] A. H. Castro Neto, F. Guinea, N. M. R. Peres, K. S. Novoselov, and A. K. Geim, The electronic properties of graphene, Rev. Mod. Phys. 81, 109 (2009).

[56] A. Kundu, H. A. Fertig, and B. Seradjeh, Floquet-Engineered Valleytronics in Dirac Systems, Phys. Rev. Lett. 116, 016802 (2016).

[57] J. W. McIver, B. Schulte, F.-U. Stein, T. Matsuyama, G. Jotzu, G. Meier, and A. Cavalleri, Light-induced anomalous Hall effect in graphene, Nat. Phys. 16, 38 (2020).

[58] E. M. Lifshitz and L. P. Pitaevskii, Statistical Physics (Butterworth-Heinemann, Stoneham, MA, 1980), Part 2.

[59] V. Vakaryuk, Stability of topological defects in chiral superconductors: London theory, Phys. Rev. B 84, 214524 (2011).

[60] V. Vadimov and M. Silaev, Predicted Nucleation of Domain Walls in $p_{x}+i p_{y}$ Superconductors by a $Z_{2}$ Symmetry-Breaking Transition in External Magnetic Fields, Phys. Rev. Lett. 111, 177001 (2013).

[61] F. C. Wu, A. H. MacDonald, and I. Martin, Theory of PhononMediated Superconductivity in Twisted Bilayer Graphene, Phys. Rev. Lett. 121, 257001 (2018).

[62] P. Stepanov, I. Das, X. Lu, A. Fahimniya, K. Watanabe, T. Taniguchi, F. H. L. Koppens, J. Lischner, L. Levitov, and D. K. Efetov, Untying the insulating and superconducting orders in magic-angle graphene, Nature (London) 583, 375 (2020).

[63] Ø. Fischer, M. Kugler, I. Maggio-Aprile, C. Berthod, and C. Renner, Scanning tunneling spectroscopy of high-temperature superconductors, Rev. Mod. Phys. 79, 353 (2007). 
[64] J. Xia, Y. Maeno, P. T. Beyersdorf, M. M. Fejer, and A. Kapitulnik, High Resolution Polar Kerr Effect Measurements of $\mathrm{Sr}_{2} \mathrm{RuO}_{4}$ : Evidence for Broken Time-Reversal Symmetry in the Superconducting State, Phys. Rev. Lett. 97, 167002 (2006).

[65] R. M. Lutchyn, P. Nagornykh, and V. M. Yakovenko, Gaugeinvariant electromagnetic response of a chiral $p_{x}+i p_{y}$ superconductor, Phys. Rev. B 77, 144516 (2008).

[66] W. Kim, F. Marsiglio, and C. S. Ting, Hall Conductivity of a Spin-Triplet Superconductor, Phys. Rev. Lett. 100, 227003 (2008).
[67] E. Taylor and C. Kallin, Intrinsic Hall Effect in a Multiband Chiral Superconductor in the Absence of an External Magnetic Field, Phys. Rev. Lett. 108, 157001 (2012).

[68] F. Yang, T. Yu, and M. W. Wu, Anomalous Hall effect in semiconductor quantum wells in proximity to chiral $p$-wave superconductors, Phys. Rev. B 97, 205301 (2018).

[69] P. M. R. Brydon, D. S. L. Abergel, D. F. Agterberg, and V. M. Yakovenko, Loop Currents and Anomalous Hall Effect from Time-Reversal Symmetry-Breaking Superconductivity on the Honeycomb Lattice, Phys. Rev. X 9, 031025 (2019). 